\title{
Distribución y conservación de especies amenazadas en Mesoamérica, Chocó y Andes tropicales
}

\section{Distribution and conservation of endangered species in Mesoamerica, Chocó and Tropical Andes}

\author{
María Cecilia Londoño-Murcia y Víctor Sánchez-Cordero* \\ Departamento de Zoología, Instituto de Biología, Universidad Nacional Autónoma de México. Apartado postal 70-153, 04510 México D. F., México. \\ *Correspondencia: victor@ibiologia.unam.mx
}

\begin{abstract}
Resumen. En este estudio se modeló el nicho ecológico proyectado como distribución potencial de 313 especies amenazadas en Mesoamérica, Chocó y los Andes tropicales, según las listas de la UICN. De estas especies, 285 fueron plantas y 28 fueron vertebrados terrestres. La superposición de las distribuciones de las especies amenazadas cubrió prácticamente toda la región. Ecuador mostró cerca del 30\% de su área con 50 especies. Colombia, Costa Rica, Guatemala, Nicaragua y Panamá mostraron $>50$, aunque en $<0.5 \%$ del área de cada país. Costa Rica, Ecuador, Nicaragua y Panamá mostraron de 21 a 50 especies en $>50 \%$ de su área. El Salvador y Honduras, de 11 a 20 en > 50\% de su área y México de 1 a 5 especies en $>50 \%$ de su área. La distribución de estas especies en áreas transformadas (agricultura y áreas urbanas) varió del 11al 30\%; El Salvador, Panamá y Guatemala mostraron $>50 \%$ de la distribución; Colombia, Honduras y México < 40\%; Belice y Ecuador, < 25\% cada uno; El Salvador, Honduras, Panamá, Nicaragua y México mostraron altos porcentajes de distribución para las clases Amphibia, Liliopsida, Polipodiopsida y los órdenes Asterales, Fabales, Laurales, Myrtales, Scrophulariales y Rubiales. En todos los países, las especies de los órdenes Campanulales y Rosales mostraron distribuciones $<35 \%$ en las áreas transformadas. En áreas naturales, la distribución de especies amenazadas protegidas varió del 12 al 19\%; la de los órdenes Fabales, Laurales, Myrtales y Rubiales fue mayor en estas áreas y menor la de los órdenes Campanulales, Asterales y la clase Liliopsida; Belice y Costa Rica mostraron la mejor representación para las clases Amphibia, Liliopsida, Polipodiopsida y los órdenes Asterales, Fabales, Laurales y Rosales. La cordillera de Talamanca en Panamá y Costa Rica, los páramos y cordilleras andinas, y la selva húmeda del oeste de Ecuador se identificaron como sitios prioritarios de conservación por su alta riqueza de especies amenazadas,
\end{abstract}

Palabras clave: modelos de nicho ecológico, especies amenazadas, distribución potencial, áreas naturales protegidas, hábitat natural, área transformada.

\begin{abstract}
This study modeled ecological niches projected as potential distributions for 313 endangered species listed in the IUCN for Mesoamerica, Chocó and Tropical Andes, of which 285 were plants, and 28 terrestrial vertebrates. Overlapping of endangered species distributions covered most of the region. Ecuador showed close to $30 \%$ of its area with 50 endangered species. Colombia, Costa Rica, Guatemala, Nicaragua y Panama showed more than 50 endangered species in $<0.5 \%$ of its area. Countries showing $>50 \%$ of its area with endangered species ( 21 to 50 species) were Costa Rica, Ecuador, Nicaragua and Panama. El Salvador and Honduras showed $>50 \%$ of its area with 11 to 20 endangered species; Mexico showed $<50 \%$ of its area with 1 to 5 endangered species. The distribution of endangered species on transformed areas (agriculture and urban settlements) ranged from 11 to 30\%. El Salvador, Panama and Guatemala showed $>50 \%$ of endangered species in transformed areas; Colombia, Honduras and Mexico showed $<40 \%$, and Belize and Ecuador showed $<25 \%$ of endangered species in transformed areas, respectively. El Salvador, Honduras, Panama, Nicaragua and Mexico showed a high proportion of endangered species in transformed areas for the Classes Amphibia, Liliopsida, Polipodiopsida, and the Orders Asterales, Fabales, Laurales, Myrtales, Scrophulariales and Rubiales. Less than $35 \%$ of endangered species occurred in transformed areas for the Orders Campanulales and Rosales. Endangered species occurring in protected areas ranged from 12 to $19 \%$; endangered species from the Orders Fabales, Laurales, Myrtales and Rubiales showed wide distributions in protected areas, while endangered species from the Orders Campanulales and Asterales, and the Class Liliopsida showed marginal distributions in protected areas. Belize and Costa Rica showed the highest representation of endangered species in protected areas. We identified the Cordillera of Talamanca in Panama and Costa Rica, the Paramos and Cordilleras of the Andes, and the tropical rainforest of Ecuador as high priority areas for holding high endangered species diversity.
\end{abstract}

Key words: ecological niche models, threatened species, potential distribution, natural protected areas, natural habitat, transformed habitat. 


\section{Introducción}

La Unión Internacional para la Conservación de la Naturaleza (UICN) ha compilado listas de especies en riesgo de extinción desde 1950, comúnmente conocidas como listas rojas. La meta es proveer un índice global del grado de degradación de la biodiversidad como consecuencia de la deforestación, e identificar las especies con mayor urgencia de conservación, con el propósito de reducir tasas de extinción (Mace et al., 2008). Las listas rojas también pretenden apoyar acciones de conservación en beneficio de especies en peligro de extinción (Fitter y Fitter, 1987). La UICN propone 3 categorías de amenaza: situación crítica (CR), amenazada (EN) y vulnerable (VU), que se definen cualitativamente por la probabilidad de extinción en un periodo determinado. Las especies clasificadas dentro de alguna de estas categorías deben cumplir al menos con 1 de 5 criterios: $a$ ), tasa alta de reducción de la población; $b$ ), área de distribución reducida, con riesgo de transformación del hábitat; c), tamaño poblacional reducido y en declive; $d$ ), tamaño poblacional muy pequeño y/o $e$ ), análisis cuantitativo de probabilidades de extinción desfavorable (Mace et al., 2008).

En este sentido, la conservación de la biodiversidad requiere un conocimiento razonable de la distribución de las especies (Margules y Sarkar, 2007). Una limitante a esta demanda es que no existe información detallada sobre la distribución geográfica de la mayoría de las especies (Graham et al., 2004; Soberón y Peterson, 2004). Recientemente se han desarrollado herramientas para producir modelos de nichos ecológicos (MNE) de las especies, proyectados como su distribución potencial (Peterson et al., 1999). Explícita o implícitamente, los MNE se basan en el concepto de Hutchinson (Hutchinson, 1957), cuya hipótesis presupone que las especies se encuentran en equilibrio con su ambiente (generalmente delimitado por características climáticas) y que la distribución se puede modelar considerando las características ambientales de los sitios donde se han observado y/o recolectado; es decir, las localidades de recolección (Guisan y Thuiller, 2005). Para definir el nicho ecológico de la especie en un espacio ambiental multidimensional (espacio ecológico), los diferentes métodos para generar MNE usan distintas reglas o algoritmos matemáticos de cómputo basados en los registros de recolección y variables ambientales. Una vez que el nicho ecológico se ha definido en un espacio ecológico, se proyecta a un espacio geográfico, produciendo un mapa de distribución potencial predictivo (Tsoar et al., 2007).

Este marco conceptual ha adquirido gran relevancia en estudios de conservación (Margules y Sarkar, 2007), específicamente en predicción de cambios de distribución en escenarios de cambio climático o predicción geográfica de una especie invasora (Rouget et al., 2004), o bien, para identificar áreas potenciales de reintroducción de especies amenazadas y en peligro de extinción (Bourg et al., 2005) o cuantificar el impacto de la pérdida de hábitat en la distribución de especies (Sánchez-Cordero et al., 2005) e identificar áreas prioritarias de conservación (Ferrier et al., 2002; Fuller et al., 200; Sarkar et al., 2009).

Este trabajo propone 2 enfoques cuantitativos usando MNE, que abordan algunos criterios de la UICN para designar el grado de amenaza de especies en las listas rojas. Dado que la deforestación, considerada como pérdida de hábitat natural, impacta negativamente la distribución de especies (Sánchez-Cordero et al. 2005) es necesario: 1), cuantificar el hábitat natural remanente en la distribución de una especie; de manera que se puedan identificar tendencias de las especies en su categorización de amenaza y, por tanto, aportar de manera objetiva su nivel de amenaza en términos de reducción de su área de distribución (Sánchez-Cordero et al., 2005; Sarkar et al., 2009) y 2), cuantificar la representatividad de las especies amenazadas (EA) en las áreas naturales protegidas (ANP). Las ANP constituyen una de las principales estrategias de conservación, por lo que es pertinente que representen adecuadamente la biodiversidad de una región y de esta manera garanticen su persistencia (Margules y Pressey, 2000).

Aunque las ANP cumplen con su papel de reducir las tasas de pérdida de hábitat (Aaron et al., 2001; Figueroa y Sánchez-Cordero, 2008; Figueroa et al., 2010), generalmente no son representativas de la biodiversidad (Rodrigues et al., 2004a). Por tanto, es importante cuantificar la representación de EA en las ANP para evaluar criterios de amenaza referentes a la proporción de su distribución en las ANP y, por ende, la probabilidad de evitar extinción poblacional regional.

La región de Mesoamérica, Andes tropicales y Chocó está reconocida como una unidad biogeográfica afín y un centro de diversidad biológica (hot spot) por su riqueza de especies y endemismos (Myers et al., 2000; Sarkar et al., 2009). Sin embargo, una tasa de deforestación alta pone en riesgo la persistencia de esta excepcional biodiversidad, resultando en un incremento notable de EA (Myers et al., 2000; Mace et al., 2008).

En este trabajo se generó MNE de una lista selecta de especies amenazadas de la IUCN que se distribuyen en la región, cuantificando los efectos negativos sobre sus distribuciones potenciales por la pérdida de hábitat natural; se evalúó la representación de las EA en las ANP, y se identificaron áreas prioritarias de conservación, por su alta riqueza de EA. 


\section{Materiales y métodos}

Área de estudio. El área de estudio incluye la región de Mesoamérica, Chocó y los Andes tropicales que conforman una unidad biogeográfica afín (Sarkar et al., 2009; Londoño et al., 2010), usando ecorregiones terrestres como criterio para delimitación (Olson et al., 2001), que consisten en 53 ecorregiones en México, Belice, Guatemala, Honduras, El Salvador, Nicaragua, Costa Rica, Panamá, Colombia y Ecuador (Fig.1). El límite norte está delineado por las ecorregiones asociadas con la depresión del Balsas en México, dada su alta afinidad biogeográfica con la biota Mesoamericana (Morrone, 2005). El límite sur está definido por la transición geográfica donde la cordillera de Los Andes se separa en 3 ramas: cordillera occidental, cordillera central y cordillera oriental. Las ecorregiones que limitan son aquellas que se interceptan con esta transición. La región de estudio se dividió en celdas de $0.02^{\circ} \times 0.02^{\circ}$, que es compatible con la escala de las coberturas ambientales de WorldClim (véase abajo), resultando en 343383 celdas con un área promedio de $4.818 \mathrm{~km}^{2}$.

Obtención de datos. Los registros de especies listadas en la UICN en las categorías de amenaza (CR, EN y VU) se obtuvieron de colecciones científicas disponibles en los siguientes sitios de internet (dirección electrónica y última fecha de consulta): MaNIS (http://manisnet.org, mayo 2010), HerpNET (http://www.herpnet.org/, mayo 2010), ORNIS (http://olla.berkeley.edu/ornisnet/, mayo 2010), REMIB (Red Mundial de Información sobre Biodiversidad; http:/www.conabio.gob.mx/remib/doctos/ remib_esp.html, mayo 2010), Smithsonian National Museum of Natural History (http://www.mnh.si.edu/rc/, abril 2007), UNIBIO (www.unibio.ibiologia.unam.mx; mayo 2010), CONABIO (http://www.biodiversidad.gob. mx/especies/especies, julio 2010) y Missouri Botanical Garden, W ${ }^{3}$ TROPICOS (http://mobot.mobot.org/W3T/ Search/vast.html, mayo 2008). Los datos climáticos se obtuvieron de WorldClim (Hijmans et al., 2005) y consistieron en 19 variables bioclimáticas, a una resolución de $1 \mathrm{~km}^{2}$. Las variables bioclimáticas se derivaron de valores mensuales de temperatura y precipitación que representan promedios anuales, estacionalidad y factores extremos y generan variables climáticas potencialmente correlacionadas estrechamente con la biología de las especies (http:// www.worldclim.org/ bioclim.htm, mayo 2010). Los datos de altitud fueron tomados de U.S. Geological Survey's Hydro-1K DEM (USGS, 1998); a partir de éstos se calculó la pendiente y la exposición, usando la extensión de análisis espacial en ArcMap 9.2.(ESRI, 2006).

Modelos de nicho ecológico. Se generaron MNE proyectados como distribuciones potenciales de 581 especies en las categorías de riesgo de la UICN, de las cuales 509 fueron plantas y 72 vertebrados terrestres, usando el programa MaxEnt versión 3.2.1 (Phillips et al., 2006). Maxent ha mostrado ser un algoritmo adecuado, resultando en modelos altamente predictivos, incluso en casos donde se cuenta con pocos registros de localidades de recolección $(<10)$ (Pearson et al., 2007). Los modelos se realizaron en formato logarítmico y usando un $25 \%$ de los datos como set de prueba.

Del total de 581 especies, sólo se incluyeron 313 en los análisis (285 especies de plantas y 28 especies de vertebrados terrestres), en virtud de que mostraron los MNE más robustos, con valores de AUC $>0.75$ (area under the curve; Phillips et al. 2006) y $P<0.05$ (Apéndice 1); estos valores indican distribuciones con alto poder predictivo (Pawar et al., 2007). Los MNE incluidos en los análisis se reclasificaron en presencias y ausencias, usando como valor umbral el valor mínimo del set de entrenamiento, apropiado para modelos que se han generado de pocos registros de recolección y teniendo un significado ecológico claro al identificar sitios ambientalmente adecuados, como son los registros de recolección (Pearson et al., 2007).

Análisis geográfico. La distribución de las 313 EA se superpusieron usando el programa ArcMap 9.2 (ESRI, 2006) y se agruparon en áreas de riqueza de especies en categorías arbitrarias: de 1 a 5, de 6 a 10, de 11 a 20, de 21 a 50 y de 51 a 116 EA, respectivamente. Este ejercicio también se realizó por separado con los grupos taxonómicos que contaron con más de 10 especies (Cuadros 1 y 2). Se calculó el área de distribución potencial, el porcentaje de hábitat transformado y el porcentaje del área de la distribución en ANP, usando el programa ArcView con la extensión Projector! y la proyección "Equal Area Cylindrical". Se consideraron hábitats transformados, las áreas de cultivo, la agricultura intensiva y extensiva y las áreas urbanas del Global Land Cover 2000; esta fuente de información es única para una misma escala que cubre toda la región de estudio (Eva et al., 2003; Latifovic et al., 2003) (Apéndice 2). Las ANP se tomaron del World Data Base on Protected Areas del 2007 (WDPA, 2007) e incluyeron todas las categorías.

\section{Resultados}

La UICN registra 1074 especies de vertebrados terrestres y 2559 especies de plantas amenazadas para la región de estudio (Mace et al., 2008). Sin embargo, sólo fue posible obtener MNE robustos para 313 especies, de las cuales 285 fueron plantas y 28 vertebrados terrestres (Cuadro 1). Estos valores representan el 2.6\% de los vertebrados terrestres y el $11 \%$ de las plantas incluidas en la lista roja de la UICN. 
Cuadro 1. Número de especies incluidas en este estudio (Análisis) y número de especies registradas en la lista roja de la UICN (Lista roja), por grupo taxonómico, en las diferentes categorías de riesgo. En el Apéndice 3, se muestran mapas para los grupos taxonómicos que presentaron más de 10 especies

\begin{tabular}{|c|c|c|c|c|c|c|c|c|}
\hline & \multirow{2}{*}{$\begin{array}{c}C R \\
\text { Análisis }\end{array}$} & \multicolumn{3}{|c|}{$E N$} & \multirow{2}{*}{$\begin{array}{c}V U \\
\text { Análisis }\end{array}$} & \multicolumn{3}{|c|}{ Total } \\
\hline & & Lista roja & Análisis & Lista roja & & Lista roja & Análisis & Lista Roja \\
\hline Animalia & 4 & 280 & 18 & 409 & 6 & 385 & 28 & 1074 \\
\hline Amphibia & 4 & 222 & 18 & 286 & 2 & 184 & 24 & 692 \\
\hline Aves & & 26 & & 62 & 1 & 112 & 1 & 200 \\
\hline Mammalia & & 19 & & 53 & 3 & 67 & & 139 \\
\hline Reptilia & & 13 & & 8 & & 22 & & 43 \\
\hline Plantae & 7 & 372 & 73 & 916 & 205 & 1271 & 285 & 2559 \\
\hline \multicolumn{9}{|l|}{ BRYOPHYTA } \\
\hline Bryopsida & & 3 & & 2 & & & & 5 \\
\hline Marchantiopsida & & 4 & & 1 & & 2 & & 7 \\
\hline \multicolumn{9}{|l|}{ LYCOPODIOPHYTA } \\
\hline Isoetopsida & & & & & & 1 & & 1 \\
\hline Lycopodiopsida & & & 1 & 2 & 3 & 8 & 4 & 10 \\
\hline Sellaginellopsida & & & & & & 1 & & 1 \\
\hline \multicolumn{9}{|l|}{ POLYPODIOPHYTA } \\
\hline Polypodiopsida & & 24 & 3 & 22 & 11 & 50 & 14 & 96 \\
\hline \multicolumn{9}{|l|}{ TRACHEOPHYTA } \\
\hline Coniferopsida & & 1 & & 6 & 1 & 9 & 1 & 16 \\
\hline Cycadopsida & & 19 & & 18 & & 17 & & 54 \\
\hline Liliopsida & 1 & 34 & 11 & 114 & 24 & 191 & 36 & 339 \\
\hline Magnoliopsida & 6 & 287 & 58 & 751 & 166 & 992 & 230 & 2030 \\
\hline Total & 11 & 652 & 91 & 1325 & 211 & 1656 & 31 & 3633 \\
\hline
\end{tabular}

CR, severamente amenazada; EN, amenazada; VU, vulnerable.

Cuadro 2. Órdenes de la clase Magnoliopsida, con el número de especies con MNE proyectados como distribuciones potenciales. Los órdenes en negritas indican aquellos para los que se presentan mapas de distribucion potencial y análisis por país

\begin{tabular}{cccc}
\hline Orden & $\begin{array}{c}\text { Núm. } \\
\text { especies }\end{array}$ & Orden & $\begin{array}{c}\text { Núm. } \\
\text { especies }\end{array}$ \\
\hline Apiales & 8 & Fagales & 4 \\
Asterales & 12 & Gentianales & 7 \\
Campanulales & 11 & Geraniales & 4 \\
Capparales & 6 & Juglandales & 2 \\
Celastrales & 3 & Lamiales & 5 \\
Cornales & 1 & Laurales & 11 \\
Dipsacales & 2 & Lecythidales & 7 \\
Ebenales & 8 & Magnoliales & 4 \\
Ericales & 1 & Malvales & 5 \\
Euphorbiales & 3 & Myrtales & 32 \\
Fabales & 17 & Piperales & 3 \\
Fagales & 4 & Polygalales & 3 \\
Gentianales & 7 & Proteales & 1 \\
Geraniales & 4 & Rosales & 12 \\
Juglandales & 2 & Rubiales & 11 \\
Lamiales & 5 & Santalales & 1 \\
Laurales & 11 & Sapindales & 9 \\
Lecythidales & 7 & Scrophulariales & 22 \\
Magnoliales & 4 & Solanales & 4 \\
Malvales & 5 & Theales & 5 \\
Myrtales & 32 & Urticales & 2 \\
Piperales & 3 & Violales & 4 \\
\hline
\end{tabular}

Al superponer las áreas de distribución de las 313 especies se observó que prácticamente se cubre toda el área de estudio (Fig. 2); sólo el $0.5 \%$, correspondiente a 8218 $\mathrm{km}^{2}$, no mostró presencia de EA. Ecuador mostró cerca del $30 \%$ de su área con una alta riqueza de especies, sumando más de 50 especies. Colombia, Costa Rica, Guatemala, Nicaragua y Panamá también presentaron más de 50 EA, aunque el porcentaje de área de cada país fue menor del 0.5\% (Fig.3). Costa Rica, Ecuador, Nicaragua y Panamá mostraron más del 50\% de su área con riqueza de EA (de 21 a 50). El Salvador y Honduras presentaron más del 50\% de su área con 11 a 20 EA; en tanto, México mostró más del 50\% de su área, con 1 a 5 EA (Fig. 3).

En áreas transformadas, El Salvador y Panamá mostraron más del 50\% del área de EA con una riqueza de 11 a 20 , Guatemala, más del 50\% del área con una riqueza de 21 a 50 EA (Cuadro 3). Colombia, Honduras y México, menos del 40\%. Belice y Ecuador $<25 \%$, respectivamente (Cuadro 3). Los porcentajes de distribución de EA en áreas transformadas variaron aproximadamente del 11 al 30\%. Guatemala, El Salvador, Honduras, Panamá, Nicaragua y México presentaron los mayores porcentajes para las clases Amphibia, Liliopsida, Polipodiopsida y los órdenes Asterales, Fabales, Laurales, Myrtales, Scrophulariales y Rubiales (Apéndice 3a,b,c,d,f,g,h,j,k). En todos los países, las clases Campanulales y Rosales mostraron distribuciones menores del 35\% en estas áreas (Apéndice 3e,i). 


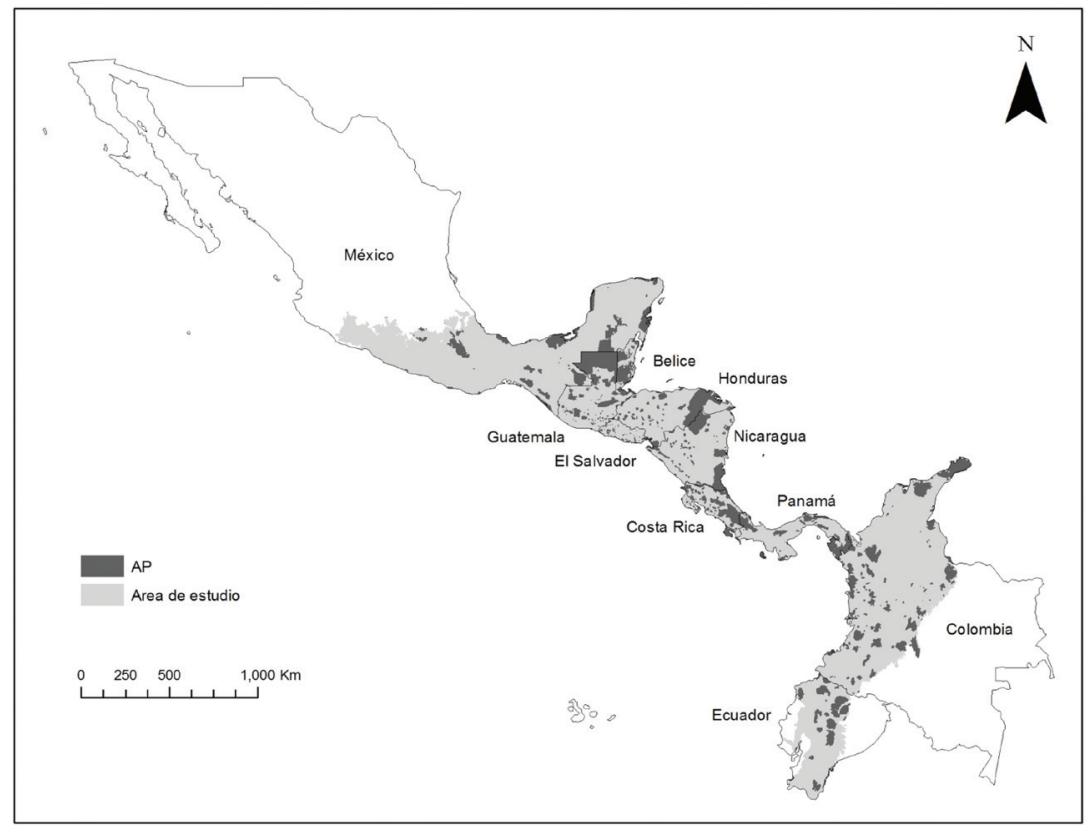

Figura 1. Área de estudio que incluye la región biogeográfica de Mesoamérica, El Chocó y los Andes tropicales.

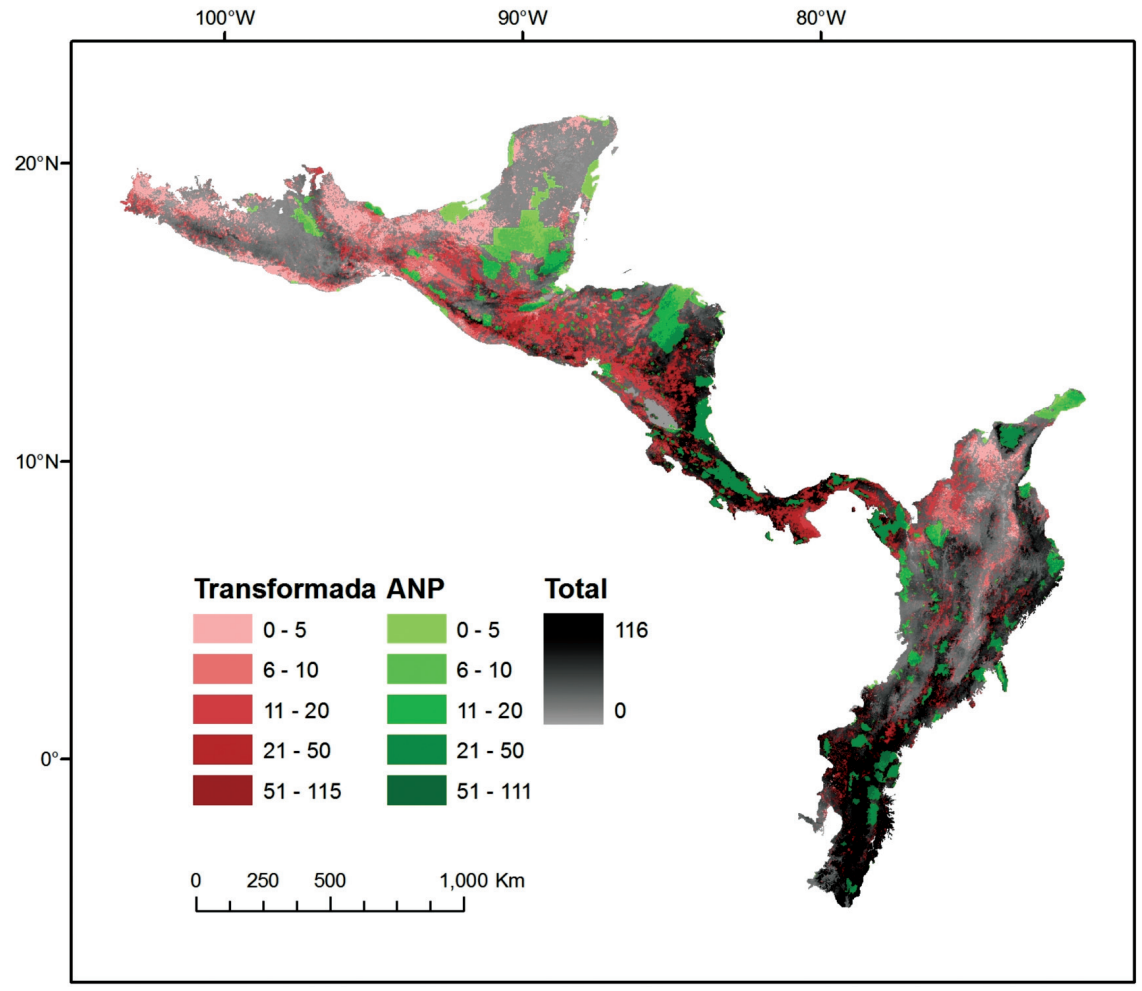

Figura 2. Concentración de riqueza de especies amenazadas en áreas transformadas (Transformada, en rojo) y en áreas naturales protegidas (ANP, en verde), al superponer la distribución potencial de 313 especies que incluyeron 28 vertebrados terrestres y 285 plantas. Se indica la riqueza de especies amenazadas, incluidas en el análisis, en las áreas con vegetación natural remanente (Total: número alto,de especies, negro; número bajo de especies, gris tenue). 


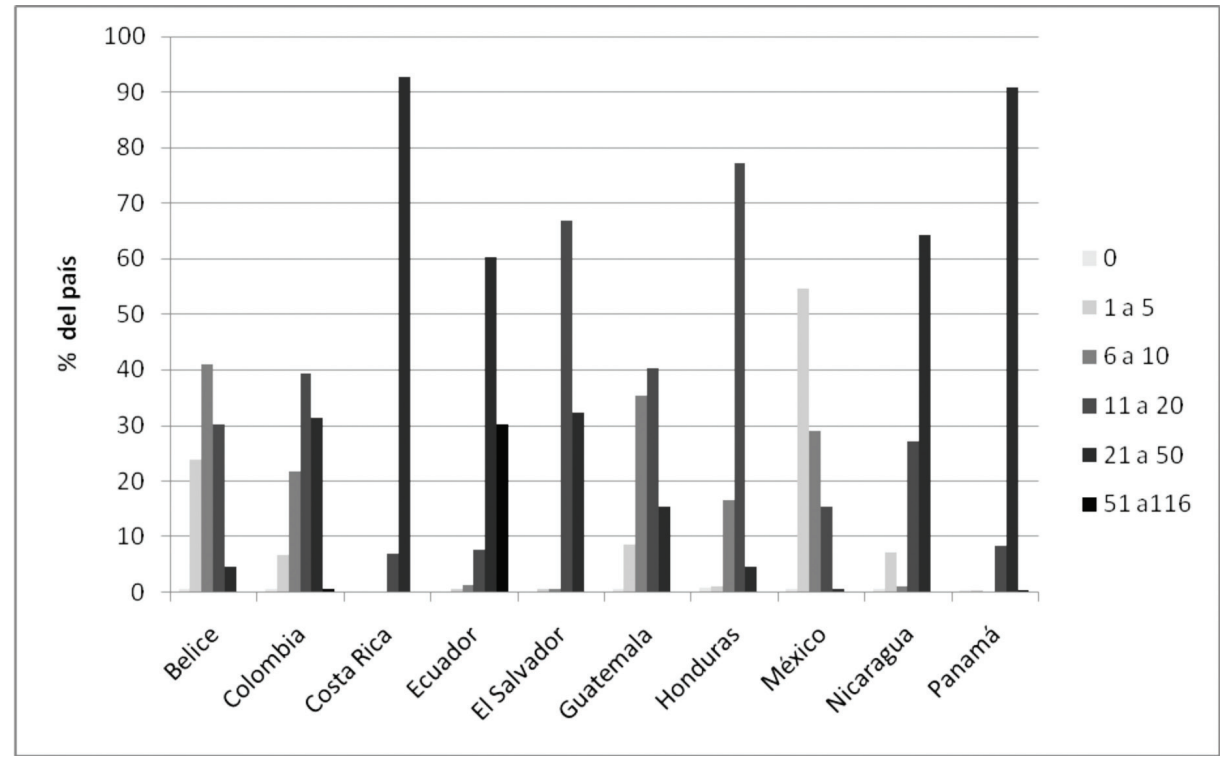

Figura 3. Porcentaje del área total del país que presenta distribución potencial de especies amenazadas para los grupos con riqueza de especies.

Cuadro 3. Porcentaje de la distribución potencial que coincide con áreas transformadas en los diferentes grupos de riqueza de especies amenazadas en cada país de la región de estudio

\begin{tabular}{lcccccc}
\hline \multicolumn{7}{c}{ Grupos de riqueza de especies } \\
& 0 & $1-5$ & $6-10$ & $11-20$ & 21 a 50 & 51 a 115 \\
\hline Belice & 3.75 & 15.28 & 18.69 & 5.60 & 1.14 & \\
Colombia & 16.55 & 34.28 & 25.69 & 16.22 & 11.28 & 26.42 \\
Costa Rica & 53.18 & 0.09 & 19.81 & 48.00 & 18.31 & \\
Ecuador & 25.96 & 15.11 & 4.72 & 7.42 & 9.67 & 7.09 \\
El Salvador & & 6.52 & 24.11 & 60.14 & 47.58 & \\
Guatemala & 2.06 & 15.65 & 21.33 & 43.90 & 55.40 & 41.27 \\
Honduras & 2.14 & 10.64 & 36.95 & 31.41 & 28.75 & \\
México & 25.97 & 30.16 & 38.53 & 37.99 & 33.68 & \\
Nicaragua & 4.71 & 0.86 & 32.65 & 48.54 & 26.25 & \\
Panamá & 36.95 & 25.43 & 24.38 & 60.87 & 38.80 & 8.24 \\
\hline
\end{tabular}

Los porcentajes de distribución potencial de las EA en las ANP variaron aproximadamente del 12 al $19 \%$ (Cuadro 5). Belice mostró los valores más altos; más de 11 EA con una distribución mayor al 60\%. Panamá, Nicaragua y Costa Rica mostraron las áreas con mayor riqueza de EA, con más del $50 \%$ de su distribución en sus respectivas ANP; Colombia, México, Ecuador y El Salvador, los valores más bajos en sus respectivas ANP. Ninguna categoría de EA mostró valores de representación en ANP mayores al 20\% (Cuadro 4).

La representación taxonómica en las ANP se cuantificó en grupos taxonómicos que incluyeron más de 10 EA: las clases Amphibia, Polypodiopsida y Liliopsida y los órdenes Asterales, Campanulales, Fabales, Laurales, Myrtales, Rosales, Rubiales y Scrophulariales, de la clase
Magnoliopsida. Los grupos taxonómicos que mostraron una mayor distribución de EA en las ANP fueron los órdenes Fabales, Laurales, Myrtales y Rubiales; los de menor distribución en ANP fueron los órdenes Campanulales, Asterales y la Clase Liliopsida. Las mejores representaciónes de EA en ANP se ubicaron en Belice y Costa Rica para las clases Amphibia, Liliopsida, Polipodiopsida y los órdenes Asterales, Fabales, Laurales y Rosales (Apéndice 3a,b,c,d,f,g,i). En El Salvador, para la clases Liliopsoda y en Guatemala, para el orden Rosales, se presentaron también valores altos en la representación de EA en las ANP (Apéndice 3b,i). Los órdenes Campanulales, Myrtales, Scrophulariales y Rubiales mostraron valores de distribución menores al 31\% de EA en ANP (Apéndice 3b,h,j,k). Para el orden Rubiales, esta tendencia se excluye en Belice, para el cual, se observó un $99 \%$ de representación en las ANP(Apéndice 3k).

Para cada grupo taxonómico se identificaron sitios con alta riqueza regional de EA que coincidiera con áreas de hábitat natural remanente fuera de las ANP. Estos sitios se consideraron prioritarios para conservación y complementarios a las ANP de la región. Para los anfibios se identificó la cordillera de Talamanca entre Panamá y Costa Rica; para los órdenes Asterales, Campanulales, Myrtales, Polypodiophytas, Rosales y Scrophulariales, los páramos andinos en el Ecuador; en el orden Fabales, las selvas húmedas del noroeste de Ecuador; en los órdenes Laurales y Liliopsidas, el oeste de la cordillera de los Andes en el centro de Ecuador, y en el orden Rubiales, el este de la cordillera Real en el Ecuador (Fig. 2; Apéndice 3a-k). 
Cuadro 4. Porcentaje de la distribución potencial en cada país de la región de estudio, que coincide con los diferentes grupos de riqueza de especies amenazadas en las áreas naturales protegidas

\begin{tabular}{lcccccc}
\hline \multicolumn{7}{c}{ Grupos de riqueza de especies } \\
& 0 & 1 a 5 & 6 a 10 & 11 a 20 & 21 a 50 & 51 a 111 \\
\hline Belice & 10.01 & 25.09 & 33.46 & 65.20 & 96.89 & \\
Colombia & 1.58 & 6.63 & 8.01 & 10.62 & 14.40 & 9.90 \\
Costa Rica & 17.61 & 90.9 & 27.07 & 9.60 & 31.67 & 84.67 \\
Ecuador & & 8.60 & 0.02 & 0.64 & 14.37 & 17.75 \\
El Salvador & & 2.75 & 13.16 & 1.94 & 4.30 & \\
Guatemala & 26.48 & 65.06 & 45.78 & 18.06 & 14.26 & \\
Honduras & 50.33 & 51.98 & 26.57 & 16.94 & 25.21 & \\
México & 31.05 & 11.33 & 6.91 & 10.13 & 1.82 & \\
Nicaragua & 35.83 & 4.86 & 17.50 & 15.15 & 19.27 & 50.06 \\
Panamá & 0.00 & 4.15 & 2.06 & 3.52 & 26.16 & 70.02 \\
\hline
\end{tabular}

\section{Discusión}

Es importante resaltar que se generaron MNE proyectados como distribuciones potenciales para un porcentaje bajo de plantas y vertebrados terrestres amenazados, incluidos en la lista roja de la IUCN (Cuadros 1 y 2) y, en este sentido, el alcance de los resultados puede ser limitado. En este estudio se realiza una búsqueda exhaustiva de registros de localidades en colecciones nacionales e internacionales, así como en bases de datos de libre acceso en internet, y se confirma el número de registros limitado para la mayoría de las EA en la región. Es posible que existan registros adicionales en colecciones científicas locales, pero la falta de acceso a estos registros dificulta su disponibilidad. Es una prioridad establecer mecanismos de acceso abierto a colecciones locales y regionales para incrementar el número de registros de EA. Por otro lado, el número reducido de recolecciones de EA debe establecer prioridades de inventarios biológicos, enfatizando las observaciones sobre la presencia de EA para obtener información confiable de su distribución y, por ende, de su conservación (Sánchez-Cordero et al., 2001; Graham et al., 2004). No obstante estas limitantes, este estudio identificó sitios de concentración de un número importante de EA de flora y fauna de la IUCN para esta región (Myers et al., 2000).

Las evaluaciones del riesgo de amenaza de las especies en las listas rojas deben efectuarse en diferentes escalas (global, regional y local), ya que pueden presentar diferente grado de amenaza, como extirpación poblacional o incluso extinción (Mace et al., 2008). Una especie puede encontrarse en buen estado de conservación en un país, región o sitio y estar amenazada a escala mundial, o bien, estar libre de amenaza en el mundo o en un país, pero en riesgo de extinción regional o local (Sánchez-Cordero et al., 2005; Mace et al., 2008). Es decir, el grado de amenaza o riesgo de extinción se debe dar en un contexto geográfico
Cuadro 5. Distribución potencial de los diferentes grupos taxonómicos en la región de estudio

\begin{tabular}{lccc}
\hline & Área total $\left(\mathrm{km}^{2}\right)$ & $\%$ AT & $\%$ ANP \\
\hline Campanulales & 274075 & 11.95 & 11.92 \\
Liliopsida & 319738 & 11.39 & 13.91 \\
Asterales & 320367 & 14.68 & 16.05 \\
Rosales & 399428 & 13.81 & 16.56 \\
Polypodiophyta & 406268 & 11.96 & 12.94 \\
Amphibia & 434762 & 20.43 & 18.72 \\
Scrophulariales & 495023 & 15.55 & 14.92 \\
Rubiales & 742785 & 17.36 & 16.57 \\
Myrtales & 808800 & 23.10 & 13.66 \\
Laurales & 857474 & 29.71 & 17.86 \\
Fabales & 1139650 & 27.01 & 14.30 \\
\hline
\end{tabular}

AT, área transformada; ANP, área protegida.

(Sánchez-Cordero et al., 2005). Las tendencias indican que las EA incluidas no mostraron un grado de amenaza equivalente en los diferentes países donde se distribuyen; los grupos taxonómicos muestran diferentes niveles de amenaza a lo largo de la región (Cuadros 3-5).

El enfoque de este estudio permitió: 1), determinar el contexto geográfico de la amenaza; 2), identificar los sitios que presentan alta riqueza de EA en diferentes categorías de riesgo y 3), la inclusión de EA de grupos multitaxón y evitar en lo posible sesgos de tendencias de distribución similares, con respecto a considerar un solo grupo taxonómico (Cuadro 3; Fig. 2). Más aún, para las especies con amenaza global de extinción, se identifican áreas con mejores condiciones de hábitat natural remanente y con mejor representatividad en ANP y que, por ende, son áreas prioritarias para enfocar esfuerzos de conservación. Se demuestra que, una proporción importante de la distribución de EA coincide con áreas transformadas, lo que indica un riesgo adicional de amenaza de extinción (Cuadros 3 y 5; Apéndice 3a-k). Se ha propuesto que las especies conservan su nicho ecológico en tiempos evolutivos, lo que supone una lenta adaptación a los hábitats nuevos (Peterson et al., 1999). Si esta tesis es correcta, sugiere que las especies no pueden mantener poblaciones viables reproductivas en áreas transformadas ("hábitat nuevo" para la especie) sin inmigración de individuos de hábitats naturales remanentes (Peterson y Holt, 2003). Por tanto, los grupos taxonómicos que mostraron esta tendencia, requieren de una alta prioridad de conservación en aquellas áreas de su distribución que aún muestran hábitat natural remanente (Sánchez-Cordero et al., 2005; Sarkar et al., 2009).

Las ANP de la región de Mesoamérica, Andes tropicales y Chocó no representan adecuadamente la diversidad biológica regional (Andelman y Willig, 2003; Armenteras et al., 2003; Cue-Bar et al., 2006; Fuller et al., 2006, 2007; 
Sarkar et al., 2009). Esta tendencia se confirma con la baja representación de las EA en las ANP, donde los valores de coincidencia geográfica fluctuaron entre el 10 y el $20 \%$ (Cuadro 4-5; Apéndice 3a-k), y es posible que sea consecuencia de que los criterios de selección y decreto de varias ANP no consideraron su contenido de biodiversidad ni las EA. Una alternativa para solventar esta deficiencia de representatividad de la biodiversidad en ANP, es generar redes de áreas de conservación que incluyan ANP y áreas enfocadas al manejo de recursos y restauración ecológica (Ervin, 2003; Rodrigues et al., 2004a; Fuller et al., 2006; Margules y Sarkar, 2007; Chan y Daily, 2008; Sarkar et al., 2009). Esto es particularmente relevante, en virtud de que se ha observado una tasa de cambio de uso de suelo y vegetación más alta en sitios ubicados fuera de las ANP (Sánchez-Azofeifa et al., 2003; Figueroa y SánchezCordero, 2008, 2010). Incluso, existen ejemplos de una deficiente protección de especies en las ANP de la región. Tal es el caso del primate amenazado, Sanguinus oedipus, en Colombia, donde se establecieron ANP que entre los años 1990 y 2000 tuvieron una pérdida del hábitat natural de poco más del $70 \%$, afectando la conservación de esta especie (Miller et al., 2004). Lo anterior confirma que es necesario establecer áreas de conservación alternativas que, integradas a las ANP, formen redes de áreas de conservación interconectadas (Margules y Sarkar, 2007; Sarkar et al., 2009).

La representatividad de las EA en las ANP contrastó en los países de la región. Los valores más altos se observaron en Belice y Costa Rica y los más bajos en México, Honduras, El Salvador y Colombia, (Cuadro 4), lo que indica que en estos países se necesita establecer programas de conservación más ambiciosos, que se enfoquen a tener una representación más adecuada de EA en las áreas avocadas a la conservación. Los resultados coinciden con análisis previos de la representatividad marginal de la biodiversidad en las ANP de estos países (Calderón et al., 2004; Sarkar et al., 2009). Consecuentemente, la planeación sistemática de la conservación debe considerarse en niveles múltiples, desde el local o paisajístico hasta el global, e integrar diferentes estrategias de conservación que conformen redes de áreas de conservación (Chan y Daily, 2008).

A pesar del bajo porcentaje de las EA incluidas en los análisis, existe un sustento de que las tendencias observadas aquí coinciden con estudios previos de algunos grupos faunísticos y florísticos; por ejemplo, Colombia, Ecuador, Panamá y México están dentro de los 20 países en el mundo con más especies de plantas amenazadas (HiltonTaylor y Mittermeier, 2000). Luna et al. (2001) encuentran que para 25 especies de plantas vasculares amenazadas de México, su representación en las ANP es muy baja, dado que la mayoría de la distribución se encuentra en los bos- ques de niebla, ecosistema amenazado y poco protegido en México. De manera similar, pese a que es el país con mayor diversidad de pinos, donde el $55 \%$ son endémicos y por lo menos 20 especies están en alguna categoría de riesgo, su representación en ANP es baja y para muchas especies se carece de programas de conservación (Sánchez-González, 2008).

Bernal y Galeno (2006) discuten que el escenario de conservación de las especies de palmas (Arecaceae) que se distribuyen en los Andes de Colombia es de amenaza. Por ende, en esos países las EA requieren particular atención, dando prioridad de investigación a programas de conservación enfocados a ubicar registros y poblaciones en regiones de alta riqueza específica (Apéndice 3a-k). Un estudio en esta dirección,es el de Granados-Tochoy et al. (2007), quienes redescubrieron en Colombia, después de 200 años, la especie amenazada Solanum humboldtianum, que cuenta con un programa específico de conservación.

La situación es similar en otros grupos biológicos. En mamíferos, al menos un $25 \%$ de las especies endémicas han perdido más del $50 \%$ de su hábitat natural, especialmente en el estado de Veracruz y en la Faja Transvolcánica Mexicana (Sánchez-Cordero et al., 2005). Para las aves, el caso del quetzal, Pharomachrus mocinno, es emblemático; Solórzano et al (2003), basándose en un estudio en México, muestran que por pérdida de hábitat natural, la distribución de esta ave ha disminuido en un $82 \%$ en los últimos 30 años, y concluyen que se necesitan esfuerzos de conservación en toda la región Mesoamericana, pues la situación de pérdida de hábitat es similar en Nicaragua, El Salvador, Guatemala y Honduras. Otra especie de ave amenazada, afectada por la transformación de los bosques de pino y encino en áreas de cultivo y ganadería en Honduras y Guatemala, es Dendroica chrysoparia (Rappole et al., 2000). Los ecosistemas de pino-encino en la región Mesoamericana contienen composición de EA que los hacen prioritarios en la selección de áreas de conservación (Sarkar et al., 2009).

El estudio de Solórzano et al. (2003) coincide con las tendencias observadas en nuestro estudio; Belice, Costa Rica y Panamá fueron los países en Mesoamérica que presentaron una mayor proporción de EA dentro de las ANP. Sin embargo, aún en estos países, hay especies que se encuentran bajo un alto riesgo de extinción, como el anfibio Craugastor punctariolus en Panamá, donde en un lapso breve se extinguieron varias poblaciones por la presencia del hongo Batrachochytrium dendrobatidis (Ryan et al., 2008). Algunas EA también presentan condiciones de conservación críticas, como el paujil, Crax alberti, en Colombia, donde se ha perdido el $96 \%$ de su hábitat natural, ubicando la especie en peligro de extinción (Melo-Vásquez et al., 2008). Ante este escenario, los MNE proyectados 
como distribuciones potenciales también pueden servir de base para tomar decisiones en beneficio de las EA. En Ecuador se generaron MNE del colibrí amenazado, Metallura baroni, y se encontraron registros de poblaciones de la especie en los sitios de distribución previstos (Tinoco et al., 2009). Asimismo, los sitios de distribución potencial de las EA pueden ser los adecuados para la reintroducción de sus poblaciones y manejo (Bourg et al., 2005).

Las iniciativas de conservación para las EA presentes en las regiones de Mesoamérica, Chocó y Andes Tropicales deben enfocarse en especies que muestran altos porcentajes de su distribución potencial en áreas transformadas de ciertos países, pero que cuenten, a su vez, con suficiente área en sitios conservados y en las ANP de otros países que ofrezcan mejores oportunidades de éxito de conservación. De esta manera, en la conservación global de EA se pueden alcanzar buenos resultados regionales.

Sin embargo, existe una diferencia entre medidas de riesgo y prioridades de conservación (Mace et al., 2008). No obstante que la UICN clasifica las especies en términos de sus riesgos de extinción, eso no equivale a determinar las prioridades de conservación, las,cuales incluyen otros factores, como costos, beneficios, logística, probabilidades de éxito y otras características biológicas de las especies (Possingham et al., 2002; Mace y Baillie, 2007). La lista roja es una herramienta para identificar casos urgentes de conservación de especies, mediante la cual se debe evaluar su situación y diseñar y establecer acciones de conservación efectivas, mas no es una herramienta para identificar prioridades de conservación per se (Mace et al., 2008).

Una crítica recurrente a la comunidad conservacionista es la inversión de altos recursos financieros en la conservación de especies en la categoría CR (situación crítica), en virtud de la supuesta baja efectividad de esta estrategia; algunas de estas especies necesitan una gran inversión de recursos y esfuerzo y los resultados son limitados. En contraste, otras especies ubicadas en otras categorías de riesgo pueden conservarse a menor costo (Mace y Baillie, 2007). La manera óptima de enfocar los esfuerzos de conservación no necesariamente se reflejan en dedicar más recursos a las especies en la categoría de mayor amenaza (Mace et al., 2008). Una alternativa es identificar sitios con alta riqueza de especies amenazadas y realizar acciones de conservación que aseguren la viabilidad de la mayor cantidad de especies amenazadas (Possingham et al., 2002; Mace y Baillie, 2007).

En este estudio se identificaron sitios prioritarios de conservación que concentran una alta riqueza de EA (Cuadros 3-5, Apéndice 3a-k). Los protocolos de priorización de proyectos y la implantación de teorías para la toma de decisiones se han empleado para optimizar la asignación de recursos a las EA; dichos protocolos se diseñaron para que de manera simultánea se consideren, costos, beneficios (incluyendo valor de las especies) y probabilidad de éxito del manejo (Margules y Sarkar, 2007). El uso de protocolos de priorización de proyectos puede mejorar sustancialmente los resultados de la conservación de especies amenazadas, al incrementar la eficiencia y asegurar la transparencia de las decisiones de manejo (McCarthy et al., 2008; Joseph et al., 2009).

\section{Agradecimientos}

Este trabajo es parte de la tesis doctoral de María Cecilia Londoño, quien agradece al Consejo Nacional de Ciencia y Tecnología (CONACyT) por la beca otorgada (CVU 206028) y al Posgrado de Ciencias Biológicas de la Universidad Nacional Autónoma de México. Dos revisores anónimos aportaron valiosas sugerencias al manuscrito, lo que benefició su calidad de contenido y presentación. Víctor Sánchez Cordero agradece el apoyo del CONACyT para la realización de este proyecto (CONACyT Núm. 25048).

\section{Literatura citada}

Aaron, G. B., R. E. Gullison, R. E. Rice y G. A. B. da Fonseca. 2001. Effectiveness of parks in protecting tropical biodiversity. Science 291:125-128.

Andelman, S. J. y M. R. Willig. 2003. Present patterns and future prospects for biodiversity in the western hemisphere. Ecology Letters 6:818-824.

Armenteras, D., F. Gast y H. Villareal. 2003. Andean forest fragmentation and the representativeness of protected natural areas in the eastern Andes, Colombia. Biological Conservation 113:245-256.

Bernal, R. y G. Galeano. 2006. Endangerment of Colombian palms (Arecaceae): change over 18 years. Botanical Journal of the Linnean Society 151:151-163.

Bourg, N. A., W. J. McShea y D. E. Gill. 2005. Putting a CART before the search: successful habitat prediction for a rare forest herb. Ecology 86:2793-2804.

Calderón, R., T. Boucher, M. Bryer, L. Sotomayor y M. Kappelle. 2004. Setting biodiversity conservation priorities in Central America: action site selection for the development of a first portfolio. The Nature Conservancy 32. San José. Costa Rica. $254 \mathrm{p}$.

Cue-Bar, E. M., J. L. Villaseñor, J. J. Morrone y G. IbarraManríquez. 2006. Identifying priority areas for conservation in Mexican tropical deciduous forest based on tree species. Interciencia 31:712-712.

Chan, K. y G. C. Daily. 2008. The payoff of conservation investments in tropical countryside. Proceedings of the National Academy of Sciences 105:19342-19342. 
Ervin, J. 2003. Protected area assessments in perspective. BioScience 53:819-822.

ESRI. 2006. ArcMAP 9.2. Geographic information system. http:// www.esri.com; última consulta: 01.VII.2010.

Eva, H. D., E. E. de Miranda, C. M. Di Bella, V. Gond, O. Huber, M. Sgrenzaroli, S. Jones, A. Coutinho, A. Dorado, M. Guimarães, C. Elvidge, F. Achard, A. S. Belward, E. Bartholomé, A. Baraldi, G. De Grandi, P. Vogt, S. Fritz y A. Hartley. 2003. The land cover map for South America in the year 2000. Joint Research Center, European Commision. GLC2000 database; 01.VII.2010.

Ferrier, S., G. Watson, J. Pearce y M. Drielsma. 2002. Extended statistical approaches to modelling spatial pattern in biodiversity in northeast New South Wales. I. Species-level modelling. Biodiversity and Conservation 11:2275-2307.

Figueroa, F. y V. Sánchez-Cordero. 2008. Effectiveness of natural protected areas to prevent land use and land cover change in Mexico. Biodiversity and Conservation 17:3223-3240.

Figueroa, F., V. Sánchez-Cordero, J. Meave e I. Trejo. 2010. Socio-economic context of land use and land cover change in Mexican biosphere reserves. Environmental Conservation 36:180-191.

Fitter, R. and M. Fitter. (eds). 1987. The Road to Extinction. International Union for Conservation of Nature , Gland, Switzerland.

Fleishman, E., R. M. Nally y J. P. Fay. 2003. Validation tests of predictive models of butterfly occurrence based on environmental variables. Conservation Biology 17:806-806.

Fuller, T., M. Munguía, M. Mayfield, V. Sánchez-Cordero y S. Sarkar. 2006. Incorporating connectivity into conservation planning: a multi-criteria case study from central Mexico. Biological Conservation 133:131-142.

Fuller, T., V. Sánchez-Cordero, P. Illoldi-Rangel, M. Linaje y S. Sarkar. 2007. The cost of postponing biodiversity conservation in Mexico. Biological Conservation 134:593600.

Graham, C. H., S. Ferrier, F. Huettman, C. Moritz y A. T. Peterson. 2004. New developments in museum-based informatics and applications in biodiversity analysis. Trends in Ecology \& Evolution 19:497-503.

Granados-Tochoy, J. C., S. Knapp y C. I. Orozco. 2007. Solanum humboldtianum (Solanaceae): An endangered new species from Colombia rediscovered 200 years after its first collection. Systematic Botany 32:200-207.

Guisan, A. y W. Thuiller. 2005. Predicting species distribution: offering more than simple habitat models. Ecology Letters 8:993-1009.

Hijmans, R. J., S. E. Cameron, J. L. Parra, P. G. Jones y A. Jarvis. 2005. Very high resolution interpolated climate surfaces for global land areas. International Journal of Climatology 25:1965-1978.

Hilton-Taylor, C. y R. A. Mittermeier 2000. 2000 IUCN red list of threatened species. IUCN.

Hutchinson, G. E. 1957. Concluding remarks. Cold Spring Harbor Symposia on Quantitative Biology 22:415-427.

Joseph, L. N., R. F. Maloney y H. P. Possingham. 2009. Optimal allocation of resources among threatened species: a project prioritization protocol. Conservation Biology 23:328-338.

Latifovic, R., Z. Zhu, J. Chilar, J. Beaubien y R. Fraser. 2003. The land cover map for North America in the year 2000. Joint Research Center. European Commision. GLC2000 database 01.VII.2010.

Londoño-Murcía, M. C., O. Téllez-Valdés y V. Sánchez-Cordero. 2010. Environmental heterogeneity of WWF ecoregions and implications for conservation in Neotropical biodiversity hotspots. Environmental Conservation 37:114-127.

Luna, I., J. J. Morrone, O. Alcántara-Ayala y D. Espinosa 2001. Biogeographical affinities among Neotropical cloud forests. Plant Systematics and Evolution 228:229-239.

Mace, G. M. y J. E. M. Baillie. 2007. The 2010 biodiversity indicators: challenges for science and policy. Conservation Biology 21:1406-1406.

Mace, G. M., N. J. Collar, K. J. Gaston, C. Hilton-Taylor, H. R. Akçakaya, N. Leader-Williams, E. J. Milner-Gulland y S. N. Stuart. 2008. Quantification of extinction risk: IUCN's system for classifying threatened species. Conservation Biology 22:1424-1442.

Margules, C. R. y S. Sarkar 2007. Systematic conservation planning. Cambridge University Press.

McCarthy, M. A., C. J. Thompson y S. T. Garnett. 2008. Optimal investment in conservation of species. Journal of Applied Ecology 45:1428-1435.

Melo-Vasquez, I., J. M. Ochoa-Quintero, H. F. Lopez-Arevalo y P. Velasquez-Sandino. 2008. Potential habitat loss and subsistence hunting of Blue Billed Curassow (Crax alberti), a Colombian critically endangered endemic bird. Caldasia 30:161-177.

Miller, L., A. Savage y H. Giraldo. 2004. Quantifying remaining forested habitat within the historic distribution of the cottontop tamarin (Saguinus oedipus) in Colombia: implications for long-term conservation. American Journal of Primatology 64:451-457.

Morrone, J. J. 2005. Hacia una síntesis biogeográfica de México. Revista Mexicana de Biodiversidad 76:207-252.

Myers, N., R. A. Mittermeier, C. G. Mittermeier, G. A. B. da Fonseca y J. Kent. 2000. Biodiversity hotspots for conservation priorities. Nature 403:853-858.

Olson, D. M., E. Dinerstein, E. D. Wikramanayake, N. D. Burgess, G. V. N. Powell, E. C. Underwood, J. A. D’Amico, I. Itoua, H. E. Strand y J. C. Morrison. 2001. Terrestrial ecoregions of the world: a new map of life on earth. BioScience 51:933938.

Pawar, S., M. S. Koo, C. Kelley, M. F. Ahmed, S. Chaudhuri y S. Sarkar. 2007. Conservation assessment and prioritization 
of areas in Northeast India: priorities for amphibians and reptiles. Biological Conservation 136:346-361.

Pearson, R. G., C. J. Raxworthy, M. Nakamura y A. T. Peterson. 2007. Predicting species distributions from small numbers of occurrence records: a test case using cryptic geckos in Madagascar. Journal of Biogeography 34:102-117.

Peterson, A. T. y R. D. Holt. 2003. Niche differentiation in Mexican birds: using point occurrences to detect ecological innovation. Ecology Letters 6:774-782.

Peterson, A. T., J. Soberon y V. Sanchez-Cordero. 1999. Conservatism of ecological niches in evolutionary time. Science 285:1265-1265.

Phillips, S. J., R. P. Anderson y R. E. Schapire. 2006. Maximum entropy modeling of species geographic distributions. Ecological Modelling 190:231-259.

Possingham, H. P., S. J. Andelman, M. A. Burgman, R. A. Medellín, L. L. Master y D. A. Keith. 2002. Limits to the use of threatened species lists. Trends in Ecology \& Evolution 17:503-507.

Rappole, J. H., D. I. King y P. Leimgruber. 2000. Winter habitat and distribution of the endangered golden-cheeked warbler (Dendroica chrysoparia). Animal Conservation 3:45-59.

Rodrigues, A. S. L., S. J. Andelman, M. I. Bakarr, L. Boitani, T. M. Brooks, R. M. Cowling, L. D. C. Fishpool, G. A. B. da Fonseca, K. J. Gaston y M. Hoffmann. 2004a. Effectiveness of the global protected area network in representing species diversity. Nature 428:640-643.

Rodrigues, A. S. L., S. J. Andelman, M. I. Bakarr, L. Boitani, T. M. Brooks, R. M. Cowling, L. D. C. Fishpool, G. A. B. da Fonseca, K. J. Gaston, M. Hoffmann, J. S. Long, P. A. Marquet, J. D. Pilgrim, R. L. Pressey, J. Schipper, W. Sechrest, S. N. Stuart, L. G. Underhill, R. W. Waller, M. E. J. Watts y X. Yan. 2004b. Effectiveness of the global protected area network in representing species diversity. Nature 428:640-643.

Rouget, M., D. M. Richardson, J. L. Nel, D. C. Le Maitre, B. Egoh y T. Mgidi. 2004. Mapping the potential ranges of major plant invaders in South Africa, Lesotho and Swaziland using climatic suitability. Diversity and Distributions 10:475-484.

Ryan, M. J., K. R. Lips y M. W. Eichholz. 2008. Decline and extirpation of an endangered Panamanian stream frog population (Craugastor punctariolus) due to an outbreak of chytridiomycosis. Biological Conservation 141:1636-1647.

Sánchez-Azofeifa, G. A., G. C. Daily, A. S. P. Pfaff y C. Busch.
2003. Integrity and isolation of Costa Rica's national parks and biological reserves: examining the dynamics of landcover change. Biological Conservation 109:123-135.

Sánchez-Cordero, V., P. Illoldi-Rangel, M. Linaje, S. Sarkar y A. T. Peterson. 2005. Deforestation and extant distributions of Mexican endemic mammals. Biological Conservation 126:465-473.

Sánchez-Cordero, V., A. T. Peterson y P. Escalante-Pliego. 2001. El modelado de la distribución de especies y la conservación de la diversidad biológica. In Enfoques contemporáneos para el estudio de la biodiversidad, H. M. Hernández, A. N. García-Alderete, F. Álvarez y M. Ulloa (eds.). Instituto de Biología, Universidad Nacional Autónoma de México, México, D.F. p. 359-379.

Sánchez-González, A. 2008. Diversity and distribution of Mexican pines, an overview. Madera y Bosques 14:107-120.

Sarkar, S., V. Sánchez-Cordero, M. C. Londoño y T. Fuller. 2009. Systematic conservation assessment for the Mesoamerica, Chocó, and Tropical Andes biodiversity hotspots: a preliminary analysis. Biodiversity and Conservation 18:1793-1828.

Soberón, J. y A. T. Peterson. 2004. Biodiversity informatics: managing and applying primary biodiversity data. Philosophical Transactions: Biological Sciences 359:689698.

Solorzano, S., M. A. Castillo-Santiago, D. A. Navarrete-Gutiérrez y K. Oyama. 2003. Impacts of the loss of neotropical highland forests on the species distribution: a case study using resplendent quetzal an endangered bird species. Biological Conservation 114:341-349.

Tinoco, B. A., P. X. Astudillo, S. C. Latta y C. H. Graham. 2009. Distribution, ecology and conservation of an endangered Andean hummingbird: the Violet-throated Metaltail (Metallura baroni). Bird Conservation International 19:6376.

Tsoar, A., O. Allouche, O. Steinitz, D. Rotem y R. Kadmon. 2007. A comparative evaluation of presence-only methods for modelling species distribution. Diversity and Distributions 13:397-405.

USGS. 1998. GTOPO30 Global 30 arc-second digital elevation model, http://www1.gsi.go.jp/geowww/globalmap-gsi/ gtopo30/gtopo30.html; última consulta: 01.VII.2010.

WDPA. 2007. The World Database on Protected Areas, Version 2007. http://www.wdpa.org/; última consulta: XII.2008. 
Apéndice 1. Robustez de los modelos de nicho ecológico de las especies amenazadas incluidas en los análisis. $\mathrm{CR}=$ situación crítica; $\mathrm{En}=$ amenazada; $\mathrm{Vu}=$ vulnerable

\begin{tabular}{|c|c|c|c|c|c|c|c|c|}
\hline Clase & Orden & Especie & $\begin{array}{c}\text { Categoría } \\
\text { UICN }\end{array}$ & $\begin{array}{c}\text { Registros } \\
\text { usados }\end{array}$ & $\begin{array}{c}A U C \\
\text { prueba }\end{array}$ & $\begin{array}{c}\text { Valor } \\
\text { umbral }\end{array}$ & $\begin{array}{l}\text { Variable que } \\
\text { contribuyó } \\
\text { más al } \\
\text { modelo }\end{array}$ & $\begin{array}{c}\% \text { de } \\
\text { contribución }\end{array}$ \\
\hline Amphibia & Anura & Atelopus varius & $\mathrm{CR}$ & 16 & 0.965 & 0.158 & Pcoq & 27.20 \\
\hline Amphibia & Anura & Bufo aucoinae & $\mathrm{Vu}$ & 15 & 0.999 & 0.848 & Pwm & 33.90 \\
\hline Amphibia & Anura & Phyllobates vitattus & En & 5 & 1 & 0.939 & Pwm & 36.00 \\
\hline Amphibia & Anura & Plectrohyla glandulosa & En & 13 & 1 & 0.005 & min_temp & 44.90 \\
\hline Amphibia & Anura & Ptychohyla salvadorensis & En & 4 & 0.999 & 0.59 & Pcoq & 32.80 \\
\hline Amphibia & Anura & Eleutherodactylus rubrimaculatus & $\mathrm{Vu}$ & 4 & 0.999 & 0.864 & Pwm & 62.40 \\
\hline Amphibia & Anura & Rana vibicaria & $\mathrm{CR}$ & 18 & 0.998 & 0.151 & Precip & 25.30 \\
\hline Amphibia & Caudata & Bolitoglossa engelhardti & En & 21 & 0.996 & 0.475 & Pwaq & 46.80 \\
\hline Amphibia & Caudata & Bolitoglossa flavimembris & En & 16 & 1 & 0.825 & Pwaq & 47.40 \\
\hline Amphibia & Caudata & Bolitoglossa pesrubra & En & 23 & 1 & 0.13 & Pwq & 24.80 \\
\hline Amphibia & Caudata & Bolitoglossa riletti & En & 6 & 0.998 & 0.578 & Mdr & 72.20 \\
\hline Amphibia & Caudata & Bolitoglossa subpalmata & En & 24 & 0.997 & 0.127 & Altura & 22.50 \\
\hline Amphibia & Caudata & Dendrotriton bromeliacius & En & 27 & 1 & 0.904 & Pwaq & 47.20 \\
\hline Amphibia & Caudata & Lineatriton lineolus & En & 7 & 0.996 & 0.416 & Ts & 56.10 \\
\hline Amphibia & Caudata & Oedipina poelzi & En & 15 & 0.997 & 0.129 & Pcoq & 20.60 \\
\hline Amphibia & Caudata & Oedipina pseudouniformis & En & 14 & 0.97 & 0.285 & Ps & 36.20 \\
\hline Amphibia & Caudata & Oedipina uniformis & En & 41 & 0.995 & 0.099 & Ts & 20.90 \\
\hline Amphibia & Caudata & Parvimolge townsendi & En & 6 & 0.999 & 0.8 & Ts & 44.30 \\
\hline Amphibia & Caudata & Pseudoeurycea brunnata & En & 18 & 1 & 0.907 & Pwaq & 40.80 \\
\hline Amphibia & Caudata & Pseudoeurycea cochranae & En & 12 & 0.995 & 0.068 & min_temp & 56.60 \\
\hline Amphibia & Caudata & Pseudoeurycea goebeli & En & 23 & 1 & 0.056 & Pwaq & 40.90 \\
\hline Amphibia & Caudata & Pseudoeurycea juarezi & En & 15 & 0.961 & 0.734 & Altura & 31.30 \\
\hline Amphibia & Caudata & Thorius magnipes & $\mathrm{CR}$ & 6 & 0.997 & 0.553 & min_temp & 44.60 \\
\hline Amphibia & Caudata & Thorius narisovalis & $\mathrm{Cr}$ & 14 & 1 & 0.435 & min_temp & 57.60 \\
\hline Aves & Psittaciformes & Amazona finschi & $\mathrm{Vu}$ & 6 & 0.999 & 0.584 & $\overline{\text { Ps }}$ & 38.50 \\
\hline Mammalia & Chiroptera & Artibeus inopinatus & $\mathrm{Vu}$ & 3 & 0.998 & 0.661 & Pdq & 35.80 \\
\hline Mammalia & Didelphimorphia & Caluromys derbianus & $\mathrm{Vu}$ & 28 & 0.804 & 0.077 & Pcoq & 23.80 \\
\hline Mammalia & Soricomorpha & Cryptotis gracilis & $\mathrm{Vu}$ & 11 & 1 & 0.264 & Temp & 27.10 \\
\hline Lycopodiopsida & Lycopodiales & Huperzia austroecuadorica & $\mathrm{Vu}$ & 6 & 0.995 & 0.548 & Altura & 41.00 \\
\hline Lycopodiopsida & Lycopodiales & Huperzia compacta & $\mathrm{Vu}$ & 8 & 0.99 & 0.264 & Altura & 54.00 \\
\hline Lycopodiopsida & Lycopodiales & Huperzia llanganatensis & $\mathrm{Vu}$ & 6 & 0.993 & 0.371 & Altura & 50.50 \\
\hline Lycopodiopsida & Lycopodiales & Huperzia loxensis & En & 4 & 0.997 & 0.485 & Altura & 33.30 \\
\hline Polypodiopsida & Blechnales & Elaphoglossum antisanae & $\mathrm{Vu}$ & 10 & 0.993 & 0.375 & Altura & 38.20 \\
\hline Polypodiopsida & Blechnales & Elaphoglossum yatesii & $\mathrm{Vu}$ & 7 & 0.998 & 0.22 & Altura & 52.40 \\
\hline Polypodiopsida & Blechnales & Diplazium divisissimum & $\mathrm{Vu}$ & 12 & 0.991 & 0.05 & Pwaq & 37.70 \\
\hline Polypodiopsida & Blechnales & Diplazium oellgaardii & $\mathrm{Vu}$ & 11 & 0.85 & 0.562 & $\mathrm{i}$ & 31.80 \\
\hline Polypodiopsida & Cyatheales & Alsophila esmeraldensis & En & 6 & 1 & 0.884 & pwaq & 29.00 \\
\hline Polypodiopsida & Cyatheales & Cyathea palaciosii & En & 5 & 0.994 & 0.366 & ps & 69.00 \\
\hline Polypodiopsida & Cyperales & Calamagrostis aurea & $\mathrm{Vu}$ & 10 & 0.997 & 0.353 & altura & 50.40 \\
\hline Polypodiopsida & Cyperales & Calamagrostis llanganatensis & $\mathrm{Vu}$ & 4 & 1 & 0.812 & altura & 45.10 \\
\hline Polypodiopsida & Cyperales & Chusquea falcata & $\mathrm{Vu}$ & 7 & 0.994 & 0.595 & altura & 43.20 \\
\hline Polypodiopsida & Cyperales & Neurolepis elata & En & 13 & 0.958 & 0.258 & altura & 22.20 \\
\hline Polypodiopsida & Cyperales & Neurolepis laegaardii & $\mathrm{Vu}$ & 10 & 0.994 & 0.58 & altura & 22.6 \\
\hline Polypodiopsida & Cyperales & Neurolepis villosa & $\mathrm{Vu}$ & 4 & 0.998 & 0.475 & altura & 41.20 \\
\hline Polypodiopsida & Hymenophyllales & Trichomanes paucisorum & $\mathrm{Vu}$ & 6 & 0.997 & 0.671 & $\mathrm{i}$ & 36.60 \\
\hline Polypodiopsida & Polypodiales & Polypodium latissimum & $\mathrm{Vu}$ & 6 & 0.998 & 0.375 & ps & 84.20 \\
\hline Coniferopsida & Coniferales & Pinus tecunumanii & $\mathrm{Vu}$ & 39 & 0.952 & 0.092 & altura & 31.80 \\
\hline Liliopsida & Arales & Anthurium balslevii & $\mathrm{Vu}$ & 10 & 0.99 & 0.264 & pwaq & 56.60 \\
\hline
\end{tabular}


Apéndice 1. Continúa.

\begin{tabular}{|c|c|c|c|c|c|c|c|c|}
\hline Clase & Orden & Especie & $\begin{array}{c}\text { Categoría } \\
\text { UICN }\end{array}$ & $\begin{array}{l}\text { Registros } \\
\text { usados }\end{array}$ & $\begin{array}{c}A U C \\
\text { prueba }\end{array}$ & $\begin{array}{l}\text { Valor } \\
\text { umbral }\end{array}$ & $\begin{array}{l}\text { Variable que } \\
\text { contribuyó } \\
\text { más al } \\
\text { modelo }\end{array}$ & $\begin{array}{c}\% \text { de } \\
\text { contribución }\end{array}$ \\
\hline Liliopsida & Arales & Anthurium cutucuense & En & 9 & 0.933 & 0.331 & ps & 84.40 \\
\hline Liliopsida & Arales & Anthurium esmeraldense & $\mathrm{Vu}$ & 4 & 0.999 & 0.7 & pwaq & 27.40 \\
\hline Liliopsida & Arales & Anthurium gualeanum & $\mathrm{Vu}$ & 7 & 0.999 & 0.414 & pcoq & 30.90 \\
\hline Liliopsida & Arales & Anthurium magnifolium & $\mathrm{Vu}$ & 4 & 0.974 & 0.479 & pwaq & 46.80 \\
\hline Liliopsida & Arales & Anthurium pedunculare & $\mathrm{Vu}$ & 13 & 0.999 & 0.118 & pwaq & 23.50 \\
\hline Liliopsida & Arales & Anthurium rimbachii & $\mathrm{Vu}$ & 18 & 0.999 & 0.533 & pwaq & 60.30 \\
\hline Liliopsida & Arales & Anthurium subcoerulescens & $\mathrm{Vu}$ & 7 & 0.997 & 0.169 & pwaq & 59.70 \\
\hline Liliopsida & Arales & Anthurium tenuifolium & En & 5 & 1 & 0.707 & ts & 68.50 \\
\hline Liliopsida & Arales & Chlorospatha ilensis & $\mathrm{Vu}$ & 9 & 0.992 & 0.477 & ps & 38.20 \\
\hline Liliopsida & Arales & Philodendron hooveri & $\mathrm{Vu}$ & 15 & 0.971 & 0.43 & pwaq & 55.90 \\
\hline Liliopsida & Arales & Philodendron musifolium & $\mathrm{Vu}$ & 14 & 0.994 & 0.366 & ts & 50.30 \\
\hline Liliopsida & Arales & Philodendron pogonocaule & $\mathrm{CR}$ & 18 & 0.948 & 0.177 & pwaq & 49.90 \\
\hline Liliopsida & Arales & Stenospermation arborescens & En & 6 & 0.997 & 0.746 & ps & 40.60 \\
\hline Liliopsida & Arales & Xanthosoma eggersii & En & 7 & 0.998 & 0.555 & pwaq & 44.50 \\
\hline Liliopsida & Arecales & Aiphanes chiribogensis & $\mathrm{Vu}$ & 10 & 0.984 & 0.332 & ts & 55.20 \\
\hline Liliopsida & Arecales & Aiphanes grandis & En & 4 & 0.998 & 0.501 & ts & 42.80 \\
\hline Liliopsida & Arecales & Aiphanes verrucosa & En & 5 & 0.997 & 0.639 & altura & 24.90 \\
\hline Liliopsida & Arecales & Bactris coloniata & $\mathrm{Vu}$ & 16 & 0.998 & 0.539 & $\mathrm{mdr}$ & 46.20 \\
\hline Liliopsida & Bromeliales & Aechmea aculeatosepala & $\mathrm{Vu}$ & 12 & 0.973 & 0.221 & ps & 60.40 \\
\hline Liliopsida & Bromeliales & Guzmania alborosea & $\mathrm{Vu}$ & 11 & 0.999 & 0.496 & pwaq & 37.10 \\
\hline Liliopsida & Bromeliales & Guzmania fusispica & $\mathrm{Vu}$ & 9 & 0.993 & 0.487 & ts & 42.20 \\
\hline Liliopsida & Bromeliales & Guzmania hollinensis & $\mathrm{Vu}$ & 9 & 0.966 & 0.538 & ps & 88.40 \\
\hline Liliopsida & Bromeliales & Guzmania madisonii & $\mathrm{Vu}$ & 11 & 0.997 & 0.291 & ps & 58.80 \\
\hline Liliopsida & Bromeliales & Mezobromelia fulgens & En & 5 & 0.994 & 0.596 & altura & 26.80 \\
\hline Liliopsida & Bromeliales & Pitcairnia ferrell-ingramiae & $\mathrm{Vu}$ & 18 & 0.999 & 0.303 & pwaq & 38.60 \\
\hline Liliopsida & Bromeliales & Pitcairnia hirtzii & $\mathrm{Vu}$ & 9 & 0.993 & 0.291 & ps & 65.30 \\
\hline Liliopsida & Bromeliales & Puya obconica & $\mathrm{Vu}$ & 5 & 0.993 & 0.619 & pcoq & 26.30 \\
\hline Liliopsida & Bromeliales & Puya pygmaea & $\mathrm{Vu}$ & 11 & 0.983 & 0.499 & mtdq & 28.50 \\
\hline Liliopsida & Bromeliales & Racinaea tandapiana & $\mathrm{Vu}$ & 7 & 0.999 & 0.467 & ts & 56.10 \\
\hline Liliopsida & Bromeliales & Racinaea tripinnata & En & 8 & 0.996 & 0.407 & altura & 62.70 \\
\hline Liliopsida & Bromeliales & Ronnbergia campanulata & En & 6 & 0.999 & 0.36 & ps & 44.80 \\
\hline Liliopsida & Bromeliales & Tillandsia emergens & $\mathrm{Vu}$ & 9 & 0.973 & 0.323 & altura & 74.70 \\
\hline Liliopsida & Bromeliales & Tillandsia rhodosticta & $\mathrm{Vu}$ & 7 & 0.986 & 0.494 & ps & 75.50 \\
\hline Liliopsida & Liliales & Bomarea chimborazensis & En & 9 & 0.986 & 0.357 & altura & 75.30 \\
\hline Liliopsida & Liliales & Bomarea uncifolia & En & 11 & 0.98 & 0.406 & altura & 56.80 \\
\hline Magnoliopsida & Apiales & Dendropanax sessiliflorus & $\mathrm{Vu}$ & 15 & 0.962 & 0.109 & pcoq & 30.00 \\
\hline Magnoliopsida & Apiales & Oreopanax hedraeostrobilus & $\mathrm{Vu}$ & 4 & 0.995 & 0.457 & ps & 39.70 \\
\hline Magnoliopsida & Apiales & Oreopanax obscurus & $\mathrm{Vu}$ & 6 & 1 & 0.553 & ps & 40.90 \\
\hline Magnoliopsida & Apiales & Oreopanax rosei & $\mathrm{Vu}$ & 7 & 0.99 & 0.305 & pwaq & 32.70 \\
\hline Magnoliopsida & Apiales & Oreopanax sessiliflorus & $\mathrm{Vu}$ & 21 & 0.995 & 0.057 & altura & 37.20 \\
\hline Magnoliopsida & Apiales & Schefflera brenesii & $\mathrm{Vu}$ & 17 & 0.939 & 0.064 & ts & 19.50 \\
\hline Magnoliopsida & Apiales & Schefflera diplodactyla & $\mathrm{Vu}$ & 17 & 0.993 & 0.303 & pdm & 59.70 \\
\hline Magnoliopsida & Apiales & Hydrocotyle yanghuangensis & $\mathrm{Vu}$ & 6 & 0.98 & 0.523 & altura & 47.30 \\
\hline Magnoliopsida & Asterales & Aequatorium jamesonii & $\mathrm{Vu}$ & 5 & 0.996 & 0.545 & altura & 81.00 \\
\hline Magnoliopsida & Asterales & Ageratina dendroides & $\mathrm{Vu}$ & 10 & 0.999 & 0.452 & altura & 27.60 \\
\hline Magnoliopsida & Asterales & Baccharis hieronymi & $\mathrm{Vu}$ & 4 & 0.99 & 0.605 & altura & 37.70 \\
\hline Magnoliopsida & Asterales & Cronquistianthus niveus & $\mathrm{Vu}$ & 11 & 0.994 & 0.182 & ts & 28.50 \\
\hline Magnoliopsida & Asterales & Diplostephium barclayanum & $\mathrm{Vu}$ & 4 & 0.999 & 0.515 & altura & 31.00 \\
\hline Magnoliopsida & Asterales & Grosvenoria rimbachii & $\mathrm{Vu}$ & 11 & 0.971 & 0.103 & altura & 39.00 \\
\hline
\end{tabular}


Apéndice 1. Continúa.

Clase

\begin{tabular}{|c|c|}
\hline Magnoliopsida & Asterales \\
\hline Magnoliopsida & Asterales \\
\hline Magnoliopsida & Asterales \\
\hline Magnoliopsida & Asterales \\
\hline Magnoliopsida & Asterales \\
\hline Magnoliopsida & Asterales \\
\hline Magnoliopsida & Campanulales \\
\hline Magnoliopsida & Campanulales \\
\hline Magnoliopsida & Campanulales \\
\hline Magnoliopsida & Campanulales \\
\hline Magnoliopsida & Campanulales \\
\hline Magnoliopsida & Campanulales \\
\hline Magnoliopsida & Campanulales \\
\hline Magnoliopsida & Campanulales \\
\hline Magnoliopsida & Campanulales \\
\hline Magnoliopsida & Campanulales \\
\hline Magnoliopsida & Campanulales \\
\hline Magnoliopsida & Capparales \\
\hline Magnoliopsida & Capparales \\
\hline Magnoliopsida & Capparales \\
\hline Magnoliopsida & Capparales \\
\hline Magnoliopsida & Capparales \\
\hline Magnoliopsida & Capparales \\
\hline Magnoliopsida & Celastrales \\
\hline Magnoliopsida & Celastrales \\
\hline Magnoliopsida & Celastrales \\
\hline Magnoliopsida & Cornales \\
\hline Magnoliopsida & Dipsacales \\
\hline Magnoliopsida & Dipsacales \\
\hline Magnoliopsida & Ebenales \\
\hline Magnoliopsida & Ebenales \\
\hline Magnoliopsida & Ebenales \\
\hline Magnoliopsida & Ebenales \\
\hline Magnoliopsida & Ebenales \\
\hline Magnoliopsida & Ebenales \\
\hline Magnoliopsida & Ebenales \\
\hline Magnoliopsida & Ebenales \\
\hline Magnoliopsida & Ericales \\
\hline Magnoliopsida & Euphorbiales \\
\hline Magnoliopsida & Euphorbiales \\
\hline Magnoliopsida & Euphorbiales \\
\hline Magnoliopsida & Fabales \\
\hline Magnoliopsida & Fabales \\
\hline Magnoliopsida & Fabales \\
\hline Magnoliopsida & Fabales \\
\hline Magnoliopsida & Fabales \\
\hline Magnoliopsida & Fabales \\
\hline Magnoliopsida & Fabales \\
\hline Magnoliopsida & Fabales \\
\hline
\end{tabular}

Especie

Gynoxys baccharoides
Gynoxys miniphylla
Gynoxys reinaldii
Loricaria scolopendra
Mutisia microcephala
Mutisia microphylla

Burmeistera holm-nielsenii

Centropogon aequatorialis

Centropogon baezanus

Centropogon comosus

Centropogon erythraeus

Centropogon medusa

Centropogon rimbachii

Centropogon steyermarkii

Centropogon ursinus

Siphocampylus affinis

Siphocampylus ecuadoriensis

Podandrogyne brevipedunculata

Cardamine lojanensis

Draba aretioides

Draba splendens

Draba spruceana

Eudema nubigena

Ilex costaricensis

$$
\text { Ilex pallida }
$$

Ilex vulcanicola

Cornus disciflora

Viburnum divaricatum

Valeriana cernua

Pouteria calistophylla

Pouteria capacifolia

Pouteria collina

Pouteria congestifolia

Pouteria fossicola

Pouteria juruana

Pradosia montana

Symplocos fuscata

Macleania loeseneriana

Croton coriaceus

Croton elegans

Garcia nutans

Abarema killipii

Astragalus sprucei

Bauhinia pichinchensis

Coursetia gracilis

Dalbergia retusa

Inga carinata

Inga extra-nodis

Inga litoralis $\begin{array}{cccc}\text { Categoría } & \text { Registros } & \text { AUC } & \text { Valor } \\ \text { UICN } & \text { usados } & \text { prueba } & \text { umbral }\end{array}$

Variable que

contribuyó

más al

modelo

$\mathrm{Vu}$

$\mathrm{Vu}$

5

0.998

0.354

altura

$0.994 \quad 0.01$

$0.992 \quad 0.606$

$0.999 \quad 0.762$

$\begin{array}{ll}0.998 & 0.579\end{array}$

$0.999 \quad 0.547$

$0.991 \quad 0.073$

$0.997 \quad 0.35$

$0.989 \quad 0.3$

$\begin{array}{ll}0.985 & 0.555\end{array}$

$0.979 \quad 0.642$

$0.999 \quad 0.283$

$0.989 \quad 0.586$

$0.997 \quad 0.614$

$0.986 \quad 0.465$

$0.983 \quad 0.265$

$0.997 \quad 0.421$

$0.98 \quad 0.159$

$0.996 \quad 0.651$

$0.996 \quad 0.11$

$0.996 \quad 0.347$

$0.993 \quad 0.622$

$0.998 \quad 0.345$

$\begin{array}{ll}0.997 & 0.039\end{array}$

$0.973 \quad 0.015$

$0.89 \quad 0.32$

$0.931 \quad 0.026$

$1 \quad 0.655$

$0.994 \quad 0.494$

$0.98 \quad 0.441$

$0.968 \quad 0.588$

$\begin{array}{lll}0.989 & 0.083\end{array}$

$0.957 \quad 0.146$

$0.929 \quad 0.212$

$0.98 \quad 0.218$

$1 \quad 0.647$

$0.996 \quad 0.507$

$0.987 \quad 0.109$

$0.985 \quad 0.203$

$0.986 \quad 0.476$

$0.914 \quad 0.024$

$0.991 \quad 0.198$

$0.994 \quad 0.429$

$0.991 \quad 0.079$

$\begin{array}{ll}1 & 0.371\end{array}$

$0.955 \quad 0.383$

$0.994 \quad 0.613$

$0.995 \quad 0.26$

$0.998 \quad 0.157$ altura

ts

ps

altura

altura

ts

ts

pdm

altura

altura

altura

ts

altura

temp

ps

altura

pwaq

altura

max_temp

altura

altura

altura

altura

mtweq

pwm

altura

pwaq

altura

mdr

pwaq

pcoq

pcoq

pcoq

pcoq

ps

mtweq

ts

altura

ts

ts

ps

altura

pwaq

ts

pdm

pcoq

ps

pwm
$\%$ de contribución

89.70

58.30

31.50

36.70

54.40

57.10

94.60

75.20

32.00

24.20

26.30

57.40

$$
42.70
$$

22.50

19.60

62.90

59.20

47.40

35.60

67.90

60.10

39.50

65.50

23.30

22.50

18.00

$$
38.50
$$

43.90

68.00

38.50

72.30

28.80

$$
35.50
$$

33.10

28.90

54.10

39.30

$$
32.20
$$

44.90

27.30

19.90

$$
57.40
$$

59.60

49.90

43.90

32.80

34.40

55.60

22.80 
Apéndice 1. Continúa.

\begin{tabular}{|c|c|c|c|c|c|c|c|c|}
\hline Clase & Orden & Especie & $\begin{array}{c}\text { Categoría } \\
\text { UICN }\end{array}$ & $\begin{array}{c}\text { Registros } \\
\text { usados }\end{array}$ & $\begin{array}{c}A U C \\
\text { prueba }\end{array}$ & $\begin{array}{l}\text { Valor } \\
\text { umbral }\end{array}$ & $\begin{array}{l}\text { Variable que } \\
\text { contribuyó } \\
\text { más al } \\
\text { modelo }\end{array}$ & $\begin{array}{c}\% \text { de } \\
\text { contribución }\end{array}$ \\
\hline Magnoliopsida & Fabales & Inga mortoniana & En & 19 & 0.926 & 0.24 & pcoq & 37.00 \\
\hline Magnoliopsida & Fabales & Inga mucuna & $\mathrm{Vu}$ & 18 & 0.999 & 0.069 & $\mathrm{mdr}$ & 32.60 \\
\hline Magnoliopsida & Fabales & Lennea viridiflora & $\mathrm{Vu}$ & 12 & 0.978 & 0.213 & altura & 32.50 \\
\hline Magnoliopsida & Fabales & Lonchocarpus phaseolifolius & $\mathrm{CR}$ & 45 & 0.97 & 0.008 & $\mathrm{pdm}$ & 55.50 \\
\hline Magnoliopsida & Fabales & Lonchocarpus phlebophyllus & En & 33 & 0.979 & 0.051 & pdm & 36.90 \\
\hline Magnoliopsida & Fabales & Lonchocarpus retiferus & En & 22 & 0.942 & 0.04 & ts & 48.50 \\
\hline Magnoliopsida & Fabales & Lupinus nubigenus & En & 6 & 1 & 0.698 & altura & 48.30 \\
\hline Magnoliopsida & Fabales & Swartzia haughtii & $\mathrm{Vu}$ & 26 & 0.851 & 0.031 & pwaq & 53.10 \\
\hline Magnoliopsida & Fabales & Swartzia oraria & $\mathrm{CR}$ & 13 & 0.958 & 0.229 & pcoq & 24.30 \\
\hline Magnoliopsida & Fagales & Quercus bumelioides & $\mathrm{Vu}$ & 59 & 0.994 & 0.004 & altura & 21.00 \\
\hline Magnoliopsida & Fagales & Quercus costaricensis & $\mathrm{Vu}$ & 37 & 0.991 & 0.231 & altura & 22.60 \\
\hline Magnoliopsida & Fagales & Quercus purulhana & $\mathrm{Vu}$ & 26 & 0.933 & 0.076 & ts & 29.00 \\
\hline Magnoliopsida & Fagales & Ticodendron incognitum & $\mathrm{Vu}$ & 46 & 0.977 & 0.02 & altura & 26.40 \\
\hline Magnoliopsida & Gentianales & Stemmadenia pauli & $\mathrm{Vu}$ & 16 & 1 & 0.719 & pcoq & 43.50 \\
\hline Magnoliopsida & Gentianales & Gentianella hirculus & En & 8 & 0.998 & 0.53 & altura & 50.20 \\
\hline Magnoliopsida & Gentianales & Gentianella hypericoides & $\mathrm{Vu}$ & 4 & 0.998 & 0.485 & ps & 48.30 \\
\hline Magnoliopsida & Gentianales & Gentianella hyssopifolia & $\mathrm{Vu}$ & 12 & 0.995 & 0.146 & altura & 33.80 \\
\hline Magnoliopsida & Gentianales & Gentianella jamesonii & En & 4 & 0.979 & 0.402 & altura & 91.60 \\
\hline Magnoliopsida & Gentianales & Gentianella longibarbata & En & 6 & 1 & 0.539 & altura & 49.70 \\
\hline Magnoliopsida & Gentianales & Halenia serpyllifolia & En & 4 & 0.99 & 0.676 & altura & 51.30 \\
\hline Magnoliopsida & Geraniales & Geranium chimborazense & $\mathrm{Vu}$ & 4 & 1 & 0.593 & altura & 56.40 \\
\hline Magnoliopsida & Geraniales & Geranium ecuadoriense & $\mathrm{Vu}$ & 6 & 0.999 & 0.771 & altura & 84.00 \\
\hline Magnoliopsida & Geraniales & Geranium loxense & $\mathrm{Vu}$ & 8 & 0.99 & 0.454 & altura & 31.90 \\
\hline Magnoliopsida & Geraniales & Geranium sericeum & $\mathrm{Vu}$ & 6 & 0.998 & 0.365 & altura & 39.30 \\
\hline Magnoliopsida & Juglandales & Juglans neotropica & En & 30 & 0.981 & 0.031 & altura & 39.50 \\
\hline Magnoliopsida & Juglandales & Juglans olanchana & En & 28 & 0.969 & 0.023 & ts & 23.70 \\
\hline Magnoliopsida & Lamiales & Lepechinia mutica & $\mathrm{Vu}$ & 4 & 0.997 & 0.569 & ts & 43.00 \\
\hline Magnoliopsida & Lamiales & Aegiphila monticola & En & 15 & 0.981 & 0.3 & temp & 31.70 \\
\hline Magnoliopsida & Lamiales & Aegiphila panamensis & $\mathrm{Vu}$ & 146 & 0.893 & 0.035 & ts & 25.40 \\
\hline Magnoliopsida & Lamiales & Aegiphila schimpffii & En & 4 & 0.997 & 0.645 & $\mathrm{i}$ & 26.90 \\
\hline Magnoliopsida & Lamiales & Vitex cooperi & En & 33 & 0.963 & 0.052 & pcoq & 33.40 \\
\hline Magnoliopsida & Laurales & Nectandra olida & $\mathrm{Vu}$ & 4 & 1 & 0.809 & ps & 72.30 \\
\hline Magnoliopsida & Laurales & Nectandra ramonensis & $\mathrm{Vu}$ & 13 & 0.909 & 0.302 & ts & 26.10 \\
\hline Magnoliopsida & Laurales & Ocotea benthamiana & $\mathrm{Vu}$ & 11 & 0.96 & 0.443 & ts & 26.60 \\
\hline Magnoliopsida & Laurales & Ocotea rivularis & $\mathrm{Vu}$ & 12 & 1 & 0.459 & pwm & 41.40 \\
\hline Magnoliopsida & Laurales & Ocotea rotundata & $\mathrm{Vu}$ & 6 & 0.988 & 0.612 & altura & 40.60 \\
\hline Magnoliopsida & Laurales & Persea obtusifolia & $\mathrm{Vu}$ & 13 & 0.944 & 0.167 & temp & 27.70 \\
\hline Magnoliopsida & Laurales & Persea schiedeana & $\mathrm{Vu}$ & 35 & 0.854 & 0.022 & altura & 24.90 \\
\hline Magnoliopsida & Laurales & Mollinedia ruae & $\mathrm{CR}$ & 15 & 0.994 & 0.079 & ts & 34.50 \\
\hline Magnoliopsida & Laurales & Siparuna cascada & $\mathrm{Vu}$ & 14 & 0.989 & 0.299 & ps & 67.50 \\
\hline Magnoliopsida & Laurales & Siparuna multiflora & $\mathrm{Vu}$ & 12 & 0.983 & 0.341 & pwaq & 44.70 \\
\hline Magnoliopsida & Laurales & Siparuna palenquensis & En & 8 & 0.983 & 0.36 & pwaq & 64.80 \\
\hline Magnoliopsida & Lecythidales & Couratari guianensis & $\mathrm{Vu}$ & 16 & 0.968 & 0.227 & pcoq & 41.20 \\
\hline Magnoliopsida & Lecythidales & Couratari scottmorii & $\mathrm{Vu}$ & 9 & 0.997 & 0.557 & pwm & 53.00 \\
\hline Magnoliopsida & Lecythidales & Eschweilera rimbachii & $\mathrm{Vu}$ & 16 & 0.965 & 0.338 & pwaq & 55.20 \\
\hline Magnoliopsida & Lecythidales & Eschweilera sclerophylla & $\mathrm{Vu}$ & 9 & 0.999 & 0.045 & pcoq & 66.90 \\
\hline Magnoliopsida & Lecythidales & Grias multinervia & $\mathrm{Vu}$ & 9 & 0.993 & 0.466 & $\mathrm{i}$ & 44.30 \\
\hline Magnoliopsida & Lecythidales & Gustavia dodsonii & En & 12 & 0.921 & 0.241 & pwaq & 59.90 \\
\hline Magnoliopsida & Lecythidales & Gustavia monocaulis & En & 5 & 0.979 & 0.585 & $\mathrm{mdr}$ & 50.30 \\
\hline
\end{tabular}


Apéndice 1. Continúa.

Clase

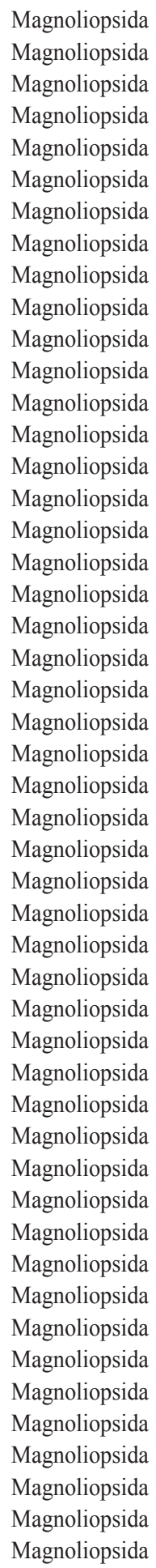

Orden

Magnoliales

Magnoliales

Magnoliales

Magnoliales

Malvales

Malvales

Malvales

Malvales

Malvales

Myrtales

Myrtales

Myrtales

Myrtales

Myrtales

Myrtales

Myrtales

Myrtales

Myrtales

Myrtales

Myrtales

Myrtales

Myrtales

Myrtales

Myrtales

Myrtales

Myrtales

Myrtales

Myrtales

Myrtales

Myrtales

Myrtales

Myrtales

Myrtales

Myrtales

Myrtales

Myrtales

Myrtales

Myrtales

Myrtales

Myrtales

Myrtales

Piperales

Piperales

Piperales

Polygalales

Polygalales

Polygalales

Proteales

Rosales
Especie

Anaxagorea phaeocarpa
Cymbopetalum torulosum
Rollinia pachyantha
Stenanona panamensis
Bombacopsis quinata
Huberodendron patinoi
Matisia grandifolia

Quararibea gomeziana

Quararibea pterocalyx

Terminalia bucidoides

Axinaea pauciflora

Axinaea sclerophylla

Axinaea sessilifolia

Blakea eriocalyx

Blakea hispida

Blakea oldemanii

Blakea rotundifolia

Brachyotum benthamianum

Brachyotum gleasonii

Brachyotum gracilescens

Brachyotum harlingii

Brachyotum jamesonii

Brachyotum johannes-julii

Brachyotum rugosum

Bucquetia nigritella

Graffenrieda harlingii

Meriania furvanthera

Miconia bolivarensis

Miconia caelata

Miconia cajanumana

Miconia dapsiliflora

Miconia dodsonii

Miconia explicita

Miconia ledifolia

Miconia pernettifolia

Miconia stenophylla

Miconia suborbicularis

Tibouchina gleasoniana

Triolena pedemontana

Eugenia salamensis

Fuchsia harlingii

Hedyosmum purpurascens

Peperomia persulcata

Piper sodiroi

Spachea correae

Monnina equatoriensis

Monnina loxensis

Euplassa occidentalis

Licania longicuspidata $\begin{array}{cccc}\text { Categoría } & \text { Registros } & \text { AUC } & \text { Valor } \\ \text { UICN } & \text { usados } & \text { prueba } & \text { umbral }\end{array}$

Variable que

contribuyó

más al

$\%$ de

modelo

En

0.989

0.104

pdm

33.30

$\mathrm{Vu}$

$0.884 \quad 0.106$

pcoq

29.60

$0.756 \quad 0.386$

pcoq

75.00

$\begin{array}{llll}\text { En } & 4 & 1 & 0.889\end{array}$

$0.938 \quad 0.041$

$0.937 \quad 0.26$

$0.99 \quad 0.497$

$1 \quad 0.912$

$0.971 \quad 0.204$

$0.991 \quad 0.188$

$0.986 \quad 0.516$

$0.995 \quad 0.363$

$0.976 \quad 0.511$

$0.948 \quad 0.261$

$0.952 \quad 0.293$

$\begin{array}{ll}0.997 & 0.221\end{array}$

$0.945 \quad 0.055$

$0.999 \quad 0.454$

$0.983 \quad 0.552$

$0.991 \quad 0.102$

$0.976 \quad 0.297$

$0.992 \quad 0.118$

$0.996 \quad 0.617$

$0.991 \quad 0.68$

$0.989 \quad 0.548$

$0.991 \quad 0.401$

$0.995 \quad 0.558$

$1 \quad 0.45$

$0.991 \quad 0.606$

$0.989 \quad 0.604$

$0.999 \quad 0.584$

$0.994 \quad 0.525$

$0.993 \quad 0.273$

$\begin{array}{ll}0.995 & 0.357\end{array}$

$0.996 \quad 0.408$

$\begin{array}{ll}0.992 & 0.437\end{array}$

$0.993 \quad 0.534$

$0.991 \quad 0.444$

$0.994 \quad 0.065$

$0.868 \quad 0.402$

$0.994 \quad 0.541$

$0.994 \quad 0.493$

$0.96 \quad 0.11$

$0.999 \quad 0.421$

$0.977 \quad 0.276$

$0.994 \quad 0.55$

$0.999 \quad 0.399$

$0.98 \quad 0.455$

$0.996 \quad 0.395$ ps

pcoq

47.80

23.30

ts

34.80

pwaq

ps

mdr

pcoq

ps

altura

pcoq

ts

ps

ts

ts

altura

altura

altura

ps

altura

altura

pcoq

pwaq

altura

i

ts

pwm

altura

ts

altura

pwaq

altura

altura

pwaq

altura

ts

pwaq

ts

altura

altura

altura

ts

altura

altura

ts

ps

ps
45.80

42.50

35.90

32.50

40.50

47.40

44.50

85.70

62.90

67.10

38.50

40.90

59.80

32.80

37.80

31.30

19.80

52.40

34.10

27.40

48.10

21.40

31.80

75.00

31.30

57.40

48.00

35.90

34.10

40.20

48.90

33.00

43.10

38.00

30.00

31.90

58.30

21.70

45.80

25.20

77.60

26.00 
Apéndice 1. Continúa.

Clase

\begin{tabular}{|c|c|}
\hline Magnoliopsida & Rosales \\
\hline Magnoliopsida & Rosales \\
\hline Magnoliopsida & Rosales \\
\hline Magnoliopsida & Rosales \\
\hline Magnoliopsida & Rosales \\
\hline Magnoliopsida & Rosales \\
\hline Magnoliopsida & Rosales \\
\hline Magnoliopsida & Rosales \\
\hline Magnoliopsida & Rosales \\
\hline Magnoliopsida & Rosales \\
\hline Magnoliopsida & Rosales \\
\hline Magnoliopsida & Rubiales \\
\hline Magnoliopsida & Rubiales \\
\hline Magnoliopsida & Rubiales \\
\hline Magnoliopsida & Rubiales \\
\hline Magnoliopsida & Rubiales \\
\hline Magnoliopsida & Rubiales \\
\hline Magnoliopsida & Rubiales \\
\hline Magnoliopsida & Rubiales \\
\hline Magnoliopsida & Rubiales \\
\hline Magnoliopsida & Rubiales \\
\hline Magnoliopsida & Rubiales \\
\hline Magnoliopsida & Santalales \\
\hline Magnoliopsida & Sapindales \\
\hline Magnoliopsida & Sapindales \\
\hline Magnoliopsida & Sapindales \\
\hline Magnoliopsida & Sapindales \\
\hline Magnoliopsida & Sapindales \\
\hline Magnoliopsida & Sapindales \\
\hline Magnoliopsida & Sapindales \\
\hline Magnoliopsida & Sapindales \\
\hline Magnoliopsida & Sapindales \\
\hline Magnoliopsida & Scrophulariales \\
\hline Magnoliopsida & Scrophulariales \\
\hline Magnoliopsida & Scrophulariales \\
\hline Magnoliopsida & Scrophulariales \\
\hline Magnoliopsida & Scrophulariales \\
\hline Magnoliopsida & Scrophulariales \\
\hline Magnoliopsida & Scrophulariales \\
\hline Magnoliopsida & Scrophulariales \\
\hline Magnoliopsida & Scrophulariales \\
\hline Magnoliopsida & Scrophulariales \\
\hline Magnoliopsida & Scrophulariales \\
\hline Magnoliopsida & Scrophulariales \\
\hline Magnoliopsida & Scrophulariales \\
\hline Magnoliopsida & Scrophulariales \\
\hline Magnoliopsida & Scrophulariales \\
\hline Magnoliopsida & Scrophulariales \\
\hline Magnoliopsida & Scrophulariales \\
\hline
\end{tabular}

Especie

Weinmannia loxensis
Ribes lehmannii
Lachemilla angustata
Lachemilla rupestris
Lachemilla sprucei
Polylepis incana
Polylepis lanuginosa
Polylepis pauta
Polylepis reticulata

Polylepis weberbaueri Rubus laegaardii

Elaeagia pastoensis

Palicourea calothyrsus

Palicourea canarina

Palicourea corniculata

Palicourea jaramilloi

Palicourea lobbii

Palicourea prodiga

Palicourea sodiroi

Psychotria fusiformis

Stilpnophyllum grandifolium Tocoyena pittieri

Dendrophthora dalstroemii Protium pittieri Cedrela fissilis

Cedrela odorata

Guarea cartaguenya

Ruagea microphylla

Swietenia humilis

Swietenia macrophylla

Zanthoxylum panamense

Guaiacum sanctum

Aphelandra attenuata

Amphitecna isthmica

Alloplectus martinianus

Columnea albiflora

Columnea capillosa

Columnea eubracteata

Columnea mastersonii

Columnea ovatifolia

Corytoplectus cutucuensis

Gasteranthus lateralis

Gasteranthus ternatus

Monopyle sodiroana

Bartsia alba

Bartsia pumila

Calceolaria adenanthera

Calceolaria dilatata

Calceolaria gossypina

$\begin{array}{cccc}\text { Categoría } & \text { Registros } & \text { VUC } & \text { Valor } \\ \text { UICN } & \text { usados } & \text { prueba umbral }\end{array}$

Variable que

más al

modelo

$\begin{array}{llll}\mathrm{Vu} & 6 & 0.984 & 0.517\end{array}$

$\mathrm{Vu}$

16

0.995

0.131

$0.991 \quad 0.372$

$\mathrm{Vu}$

$\mathrm{Vu}$

$\mathrm{Vu}$

$\mathrm{Vu}$

$0.997 \quad 0.44$

$0.999 \quad 0.355$

$0.993 \quad 0.016$

$0.878 \quad 0.26$

$0.999 \quad 0.304$

$0.876 \quad 0.115$

$0.991 \quad 0.046$

$\begin{array}{ll}0.987 & 0.49\end{array}$

$0.854 \quad 0.156$

$0.995 \quad 0.027$

$0.954 \quad 0.234$

$0.995 \quad 0.217$

$0.945 \quad 0.214$

$0.991 \quad 0.544$

$0.997 \quad 0.612$

$0.998 \quad 0.458$

$0.977 \quad 0.286$

$0.998 \quad 0.022$

$\begin{array}{ll}0.909 & 0.127\end{array}$

$0.995 \quad 0.518$

$0.974 \quad 0.064$

$\begin{array}{ll}0.986 & 0.251\end{array}$

$\begin{array}{ll}0.803 & 0.067\end{array}$

$0.971 \quad 0.304$

$0.999 \quad 0.633$

$0.952 \quad 0.036$

$0.806 \quad 0.077$

$0.945 \quad 0.086$

$\begin{array}{ll}0.916 & 0.001\end{array}$

$0.985 \quad 0.635$

$0.957 \quad 0.243$

$0.962 \quad 0.36$

$0.974 \quad 0.396$

$0.992 \quad 0.405$

$0.998 \quad 0.284$

$0.996 \quad 0.302$

$1 \quad 0.543$

$0.985 \quad 0.173$

$0.946 \quad 0.378$

$0.999 \quad 0.733$

$0.988 \quad 0.3$

$0.999 \quad 0.438$

$1 \quad 0.528$

$0.992 \quad 0.293$

$0.89 \quad 0.075$

$0.997 \quad 0.555$

$$
\text { i }
$$

altura

altura

altura

altura

altura

altura

altura

altura

altura

altura

pcoq

ts

ps

ps

altura

altura

ps

ts

ps

ps

pcoq

altura

pcoq

min_temp

ts

pwaq

ts

pdm

ts

precip

pdm

pwaq

pcoq

ps

ps

ps

ts

ts

ts

ps

ts

ts

pwaq

altura

altura

altura

ts

altura
$\%$ de contribución

24.40

53.40

49.70

50.00

87.70

61.80

35.40

53.80

69.10

40.00

55.00

53.40

44.40

68.60

40.70

49.80

53.10

32.00

70.10

91.30

74.10

34.80

24.10

28.10

26.20

27.20

33.30

26.40

33.60

25.80

27.50

29.30

64.80

32.60

37.10

46.00

63.20

47.10

47.80

61.60

77.40

48.30

48.10

59.70

77.30

41.20

57.40

38.60

95.50 
Apéndice 1. Continúa.

Clase

Magnoliopsida

Magnoliopsida

Magnoliopsida

Magnoliopsida

Magnoliopsida

Magnoliopsida

Magnoliopsida

Magnoliopsida

Magnoliopsida

Magnoliopsida

Magnoliopsida

Magnoliopsida

Magnoliopsida

Magnoliopsida

Magnoliopsida

Magnoliopsida

Magnoliopsida

Magnoliopsida

Magnoliopsida

Magnoliopsida
Orden

Scrophulariales

Scrophulariales

Scrophulariales

Scrophulariales

Scrophulariales

Solanales

Solanales

Solanales

Solanales

Theales

Theales

Theales

Theales

Theales

Urticales

Urticales

Violales

Violales

Violales

Violales
Especie

Calceolaria oxyphylla

Calceolaria pedunculata

Calceolaria serrata

Calceolaria spruceana

Calceolaria stricta

Iochroma lehmannii

Solanum dolichorhachis

Solanum interandinum

Solanum leiophyllum

Caryocar costaricense

Clusia croatii

Clusia osseocarpa

Lacunaria panamensis

Freziera minima

Cecropia longipes

Cecropia maxima

Casearia mexiae

Passiflora jamesonii

Passiflora loxensis

Passiflora roseorum

\begin{tabular}{|c|c|c|c|c|c|}
\hline $\begin{array}{c}\text { Categoría } \\
\text { UICN }\end{array}$ & $\begin{array}{c}\text { Registros } \\
\text { usados }\end{array}$ & $\begin{array}{c}\text { AUC } \\
\text { prueba }\end{array}$ & $\begin{array}{c}\text { Valor } \\
\text { umbral }\end{array}$ & $\begin{array}{l}\text { Variable que } \\
\text { contribuyó } \\
\text { más al } \\
\text { modelo }\end{array}$ & $\begin{array}{c}\% \text { de } \\
\text { contribución }\end{array}$ \\
\hline $\mathrm{Vu}$ & 7 & 0.992 & 0.379 & altura & 33.60 \\
\hline $\mathrm{Vu}$ & 12 & 0.996 & 0.059 & altura & 29.40 \\
\hline $\mathrm{Vu}$ & 5 & 0.999 & 0.502 & pcoq & 44.90 \\
\hline $\mathrm{Vu}$ & 7 & 0.977 & 0.311 & altura & 82.10 \\
\hline $\mathrm{Vu}$ & 6 & 0.995 & 0.449 & ts & 33.80 \\
\hline $\mathrm{Vu}$ & 4 & 0.999 & 0.497 & pcoq & 27.40 \\
\hline $\mathrm{Cr}$ & 4 & 0.987 & 0.312 & pwaq & 60.70 \\
\hline $\mathrm{Vu}$ & 20 & 0.99 & 0.205 & altura & 50.80 \\
\hline $\mathrm{Vu}$ & 4 & 0.971 & 0.523 & altura & 67.30 \\
\hline $\mathrm{Vu}$ & 14 & 0.76 & 0.218 & pwm & 27.70 \\
\hline $\mathrm{Vu}$ & 81 & 0.972 & 0.067 & ts & 25.70 \\
\hline $\mathrm{Vu}$ & 10 & 0.876 & 0.095 & $\mathrm{mdr}$ & 40.80 \\
\hline En & 18 & 0.997 & 0.308 & $\mathrm{i}$ & 25.00 \\
\hline $\mathrm{Vu}$ & 6 & 0.963 & 0.593 & altura & 33.80 \\
\hline En & 10 & 0.993 & 0.295 & min_temp & 43.20 \\
\hline $\mathrm{Vu}$ & 5 & 0.998 & 0.623 & ts & 67.00 \\
\hline En & 5 & 0.985 & 0.513 & altura & 50.00 \\
\hline $\mathrm{Vu}$ & 8 & 0.984 & 0.143 & altura & 77.20 \\
\hline En & 7 & 0.99 & 0.464 & altura & 41.90 \\
\hline $\mathrm{Vu}$ & 8 & 0.996 & 0.443 & altura & 44.40 \\
\hline
\end{tabular}

Apéndice 2. Clasificación de los tipos de vegetación de Global Land Cover 2000, con la correspondiente en el análisis del presente trabajo.

Clasificación de

Global Land Cover 2000

Agriculture - intensive

Bamboo dominated forest

Barren / bare soil

Burnt area (resent burnt area)

Closed deciduous forest

Closed evergreen tropical forest

Closed montane grasslands

Closed semi deciduous forest

Closed semi-humid forest

Closed shrublands

Closed steppe grasslands

Consolidated rock sparse vegetation

Cropland

Cropland and shrubland/woodland

Desert

Forest plantations (Llanos of

Venezuela)

Fresh water flooded forests

Grass savannah

Herbaceous wetlands

Mangroves
Clasificación usada

en el análisis de este estudio

áreas vegetación

vegetación natural

vegetación natural

áreas transformadas

vegetación natural

vegetación natural

áreas transformadas

vegetación natural

vegetación natural

áreas transformadas

áreas transformadas

vegetación natural

áreas transformadas

áreas transformadas

vegetación natural

áreas transformadas

vegetación natural

vegetación natural

vegetación natural

vegetación natural
Apéndice 2. Continúa

Montane forests $>1000 \mathrm{~m}$ - open semi humid

Montane forests $>1000 \mathrm{~m}$ - closed

semi -deciduous

Montane forests $>1000 \mathrm{~m}$ - open

deciduous

Montane forests $>1000 \mathrm{~m}$ - open

evergreen

Montane forests $>1000 \mathrm{~m}$ - open semi-

deciduous

Montane forests $>1000 \mathrm{~m}$ - transition

forest

Montane forests $>1000 \mathrm{~m}$ - closed

semi humid

Montane forests $>1000 \mathrm{~m}$ flooded

forest

Montane forests $>1000 \mathrm{~m}$ flooded

forest

Montane forests $>1000 \mathrm{~m}$ flooded

forest

Montane forests $>1000 \mathrm{~m}$ - bamboo dominated

Montane forests $>1000 \mathrm{~m}$ - closed

deciduous

Montane forests $>1000 \mathrm{~m}$ - closed

temperate deciduous

Montane forests $>1000 \mathrm{~m}$ - dense

evergreen

Montane forests $>1000 \mathrm{~m}$ - open

temperate deciduous vegetación natural

vegetación natural

vegetación natural

vegetación natural

vegetación natural

vegetación natural

vegetación natural

vegetación natural

vegetación natural

vegetación natural

vegetación natural

vegetación natural

vegetación natural

vegetación natural

vegetación natural 
Apéndice 2. Continúa

Montane forests $>1000 \mathrm{~m}$ - temperate mixed

Montane forests $>1000 \mathrm{~m}$ - temperate closed broadleaf

Montane forests 500-1 000 - closed

semi humid

Montane forests 500-1 000 - open semi humid

Montane forests 500-1 000 - bamboo

Montane forests 500-1 000 - dense

evergreen

Montane forests 500-1 000 - open

evergreen

Montane forests 500-1 000m - flooded

forest

Montane forests 500-1 000m - closed

deciduous

Montane forests 500-1 000m - closed

semi -deciduous

Montane forests 500-1 000m - closed

temperate deciduous

Montane forests 500-1 000m - flooded

forest

Montane forests 500-1 000m - flooded

forest

Montane forests 500-1 000m - open

deciduous

Montane forests 500-1 000m - open

semi- deciduous

Montane forests 500-1 000m - open

temperate deciduous

Montane forests 500-1 000m -

temperate mixed

Montane forests 500-1 000m -

transition forest

Montane forests $500-1000 \mathrm{~m}$

-temperate closed broadleaf

Mosaic agriculture / degraded forests

Mosaic agriculture / degraded

vegetation

Open deciduous forest

Open evergreen tropical forest

Open montane grasslands

Open semi deciduous forest

Open semi-humid forest

Open shrublands

Open steppe grasslands

Periodically flooded savannah

Permanent swamp forests

Polar Grassland with a dwarf-sparse

shrub layer

Polar Grassland with a sparse shrub layer

Semi deciduous transition forest vegetación natural

vegetación natural

vegetación natural

vegetación natural

vegetación natural

vegetación natural

vegetación natural

vegetación natural

vegetación natural

vegetación natural

vegetación natural

vegetación natural

vegetación natural

vegetación natural

vegetación natural

vegetación natural

vegetación natural

vegetación natural

vegetación natural

áreas transformadas

áreas transformadas

vegetación natural vegetación natural vegetación natural vegetación natural vegetación natural áreas transformadas vegetación natural áreas transformadas vegetación natural vegetación natural

vegetación natural

vegetación natural
Apéndice 2. Continua

Shrub Savannah

Snow and Ice

Sparse desertic steppe shrub /grasslands

Subpolar needleleaved evergreen forest open canopy - lichen understory

Temperate closed deciduous broadleaf forests

Temperate closed evergreen broadleaf

forest

Temperate mixed evergreen broadleaf

forests

Temperate open deciduous broadleaf forests

Temperate or sub-polar broadleaved deciduous forest - closed canopy Temperate or subpolar broadleaved deciduous shrubland - open canopy Temperate or subpolar broadleaved evergreen shrubland - closed canopy Temperate or subpolar grassland

Temperate or subpolar grassland with a sparse shrub layer

Temperate or subpolar grassland with a sparse tree layer

Temperate or sub-polar mixed

broaddleleaved or needleleaved forest open canopy

Temperate or sub-polar mixed

broadleaved and needleleaved dwarf-

shrubland - open canopy

Temperate or sub-polar mixed

broadleaved or needleleaved forest -

closed canopy

Temperate or sub-polar needleleaved evergreen forest - closed canopy Temperate or sub-polar needleleaved evergreen forest - open canopy Temperate or subpolar needleleaved evergreen shrubland - open canopy Temperate or sub-polar needleleaved mixed forest - closed canopy Tropical or sub-tropical broadleaved deciduous forest - closed canopy Tropical or sub-tropical broadleaved evergreen forest - closed canopy Tropical or sub-tropical broadleaved evergreen forest - open canopy Unconsolidated material sparse vegetation (old burnt or other disturbance)

Urban

Urban and built-up

Water bodies

Wetlands vegetación natural vegetación natural vegetación natural vegetación natural

vegetación natural vegetación natural

vegetación natural

vegetación natural

vegetación natural

vegetación natural

vegetación natural

vegetación natural vegetación natural

vegetación natural

vegetación natural

vegetación natural

vegetación natural

vegetación natural

vegetación natural

vegetación natural

vegetación natural

vegetación natural

vegetación natural

vegetación natural

áreas transformadas

áreas transformadas áreas transformadas vegetación natural vegetación natural 
Apéndice 3. Mapas (a- k ) en los que semuestra la superposición de la distribución potencial de los diferentes grupos taxonómicos en áreas con vegetación natural remanente (número de especies alto, negro; número de especies bajo, gris tenue; distribución potencial en áreas transformadas, rojo; áreas naturales protegidas (ANP), verde. En el cuadro inserto se muestra el área $\left(\mathrm{km}^{2}\right)$ de la distribución potencial, el porcentaje que coincide en áreas transformadas (\% transformado) y el porcentaje que coincide con áreas naturales protegidas (\%ANP), respectivamente, en los países de la región. (a) Amphibia, (b) Liliopsida, (c) Polipodiopsida, (d) Asterales, (e) Campanulales, (f) Fabales, (g) Laurales, (h) Myrtales, (i) Rosales, (j) Scrophulariales, (k) Rubiales.

\section{a}

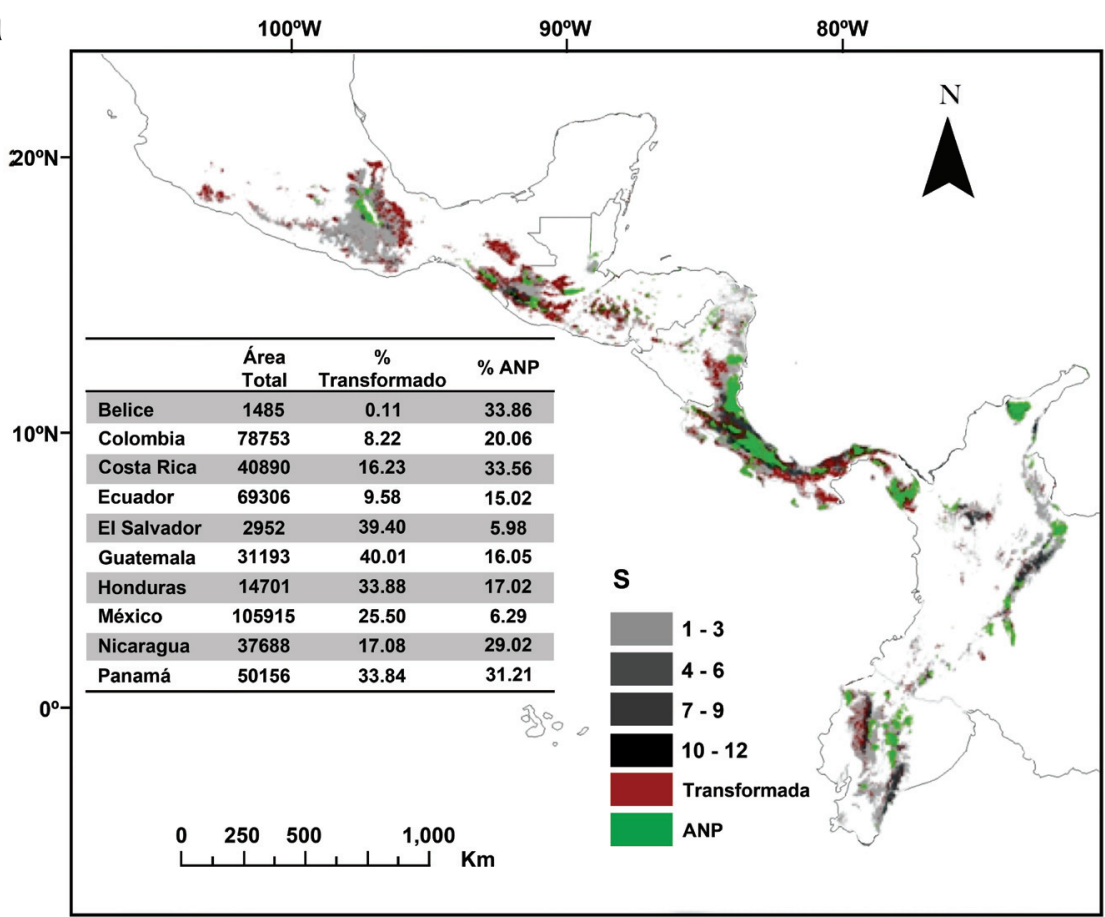

b

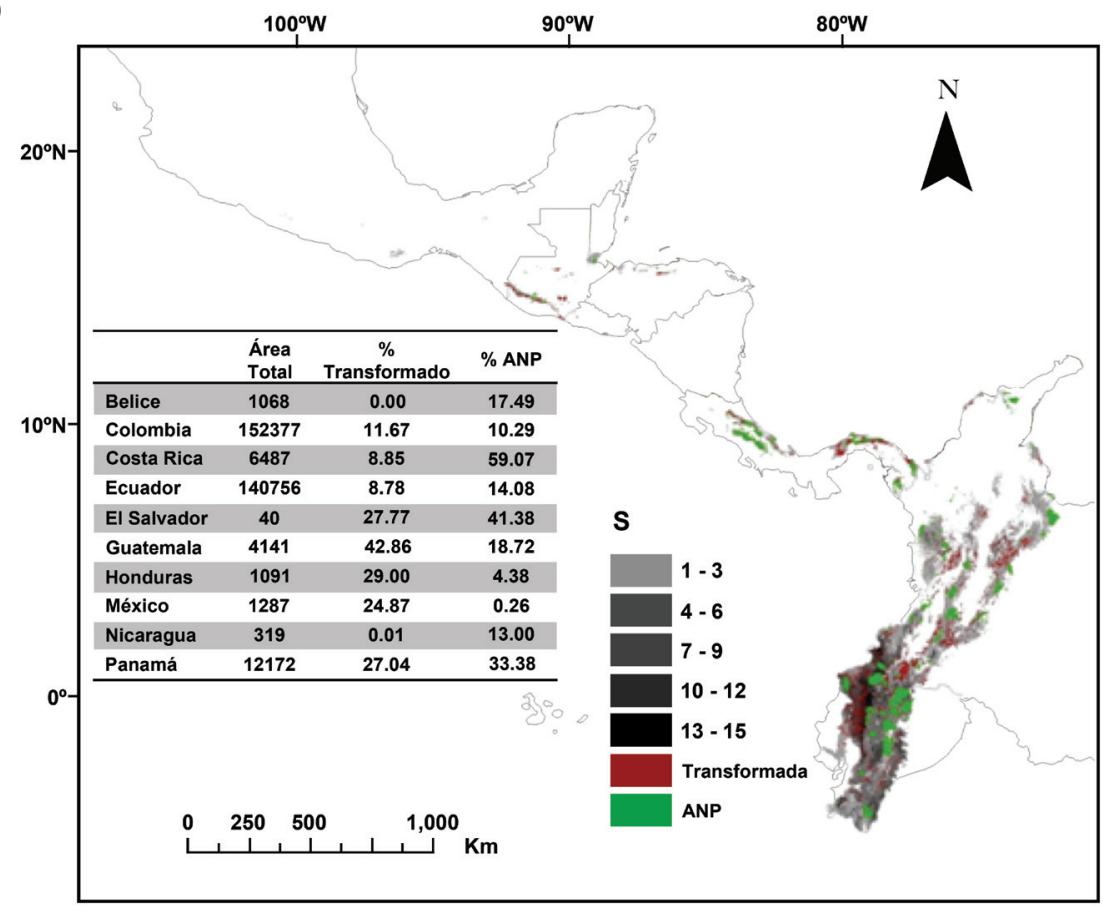


C

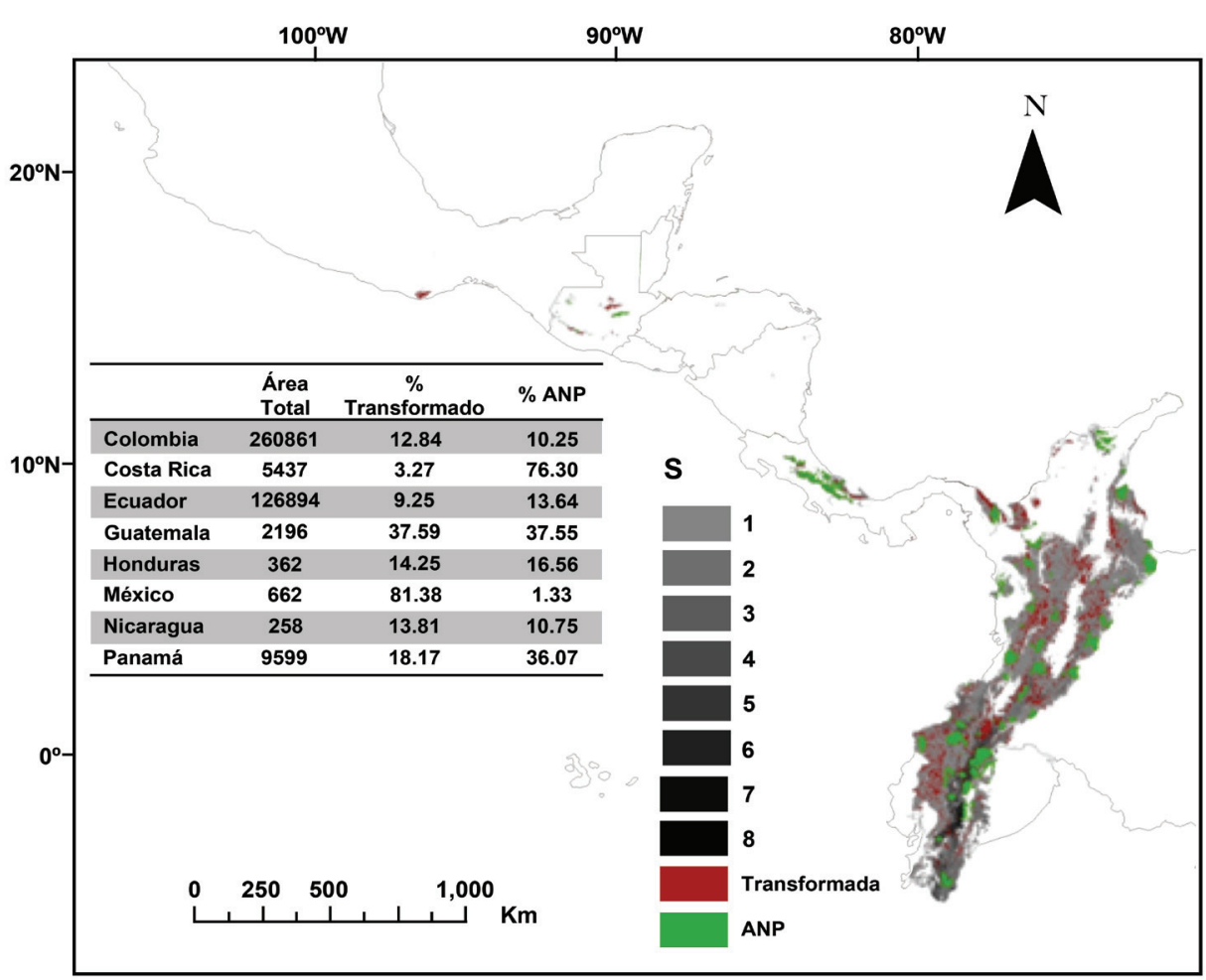

d

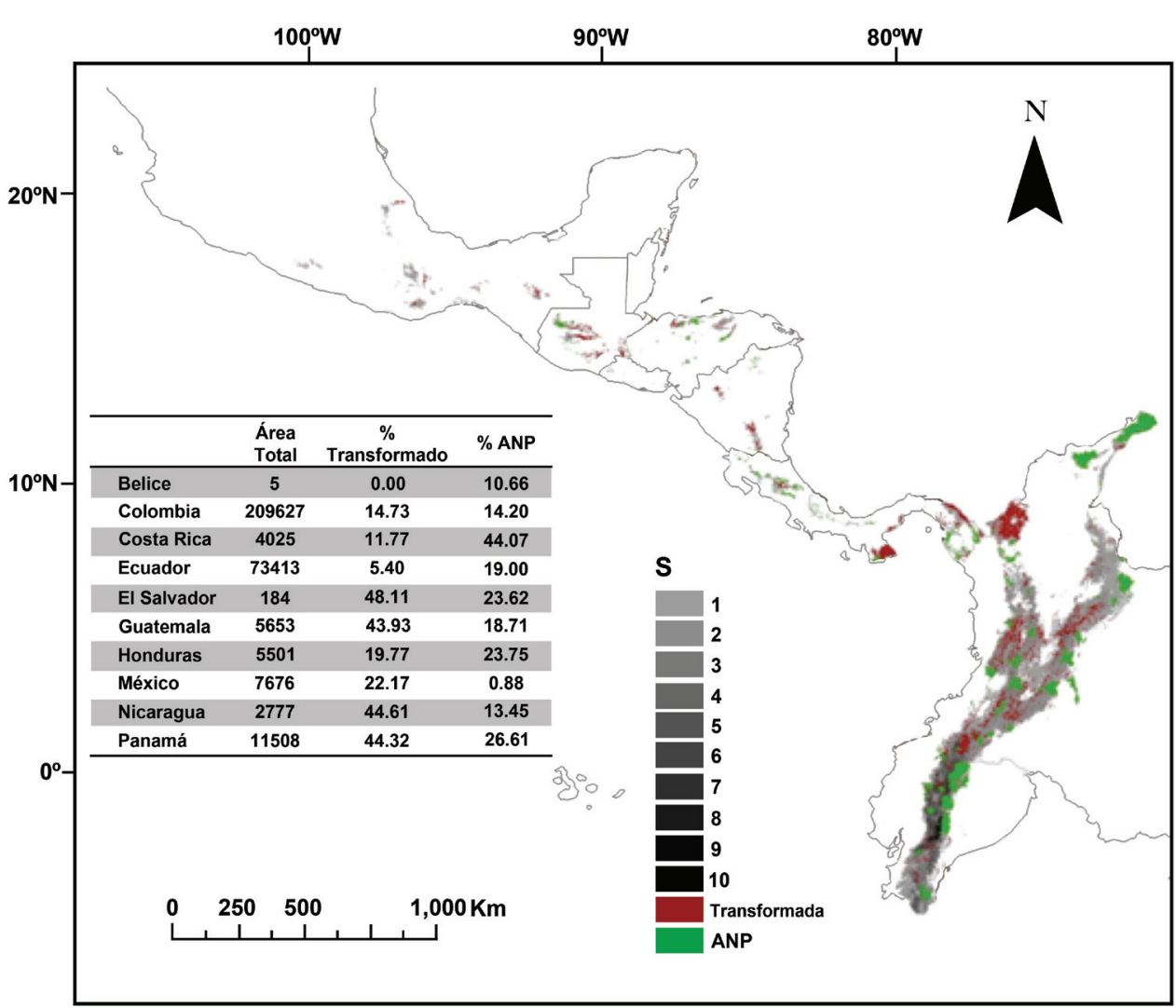


e

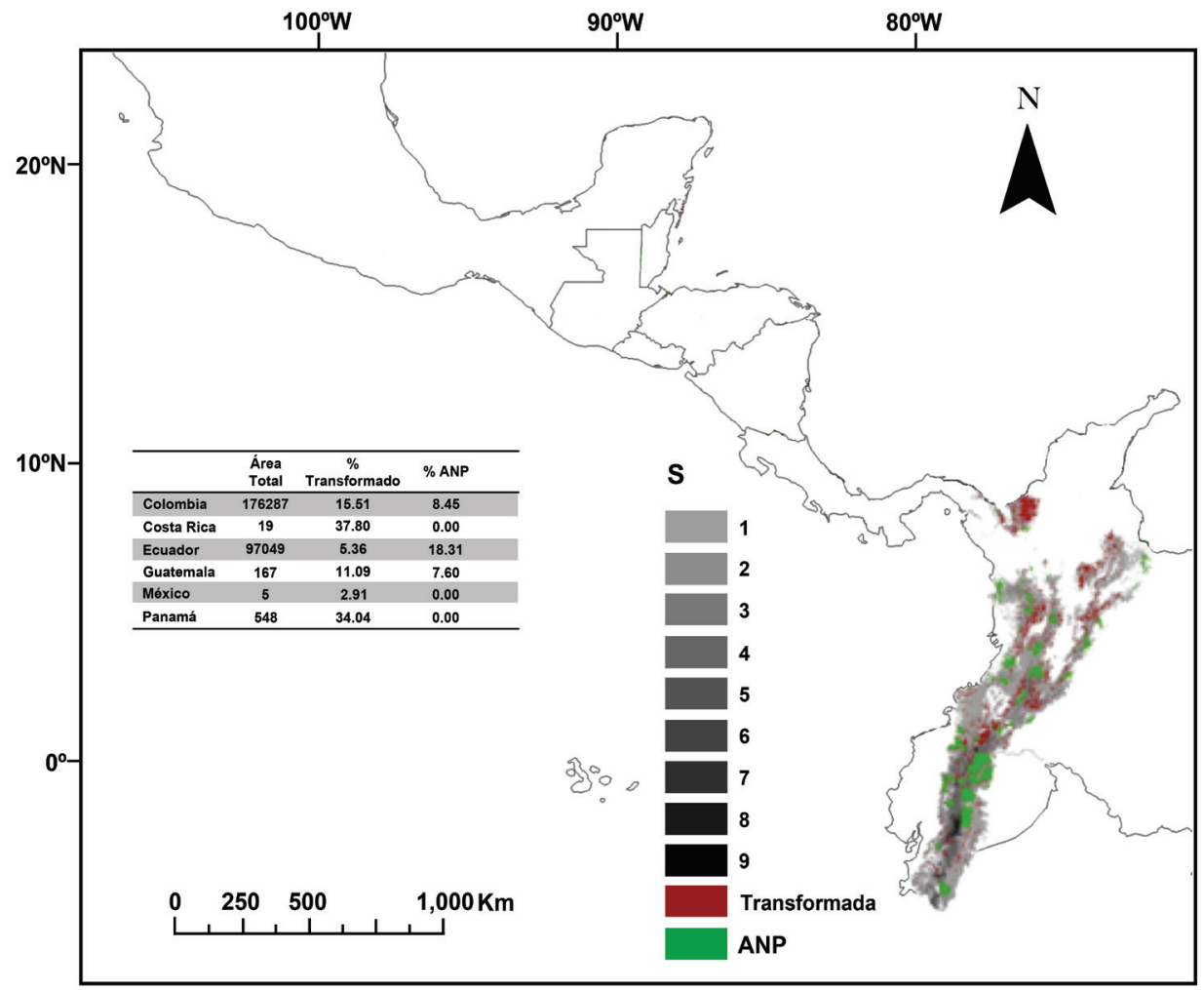

f

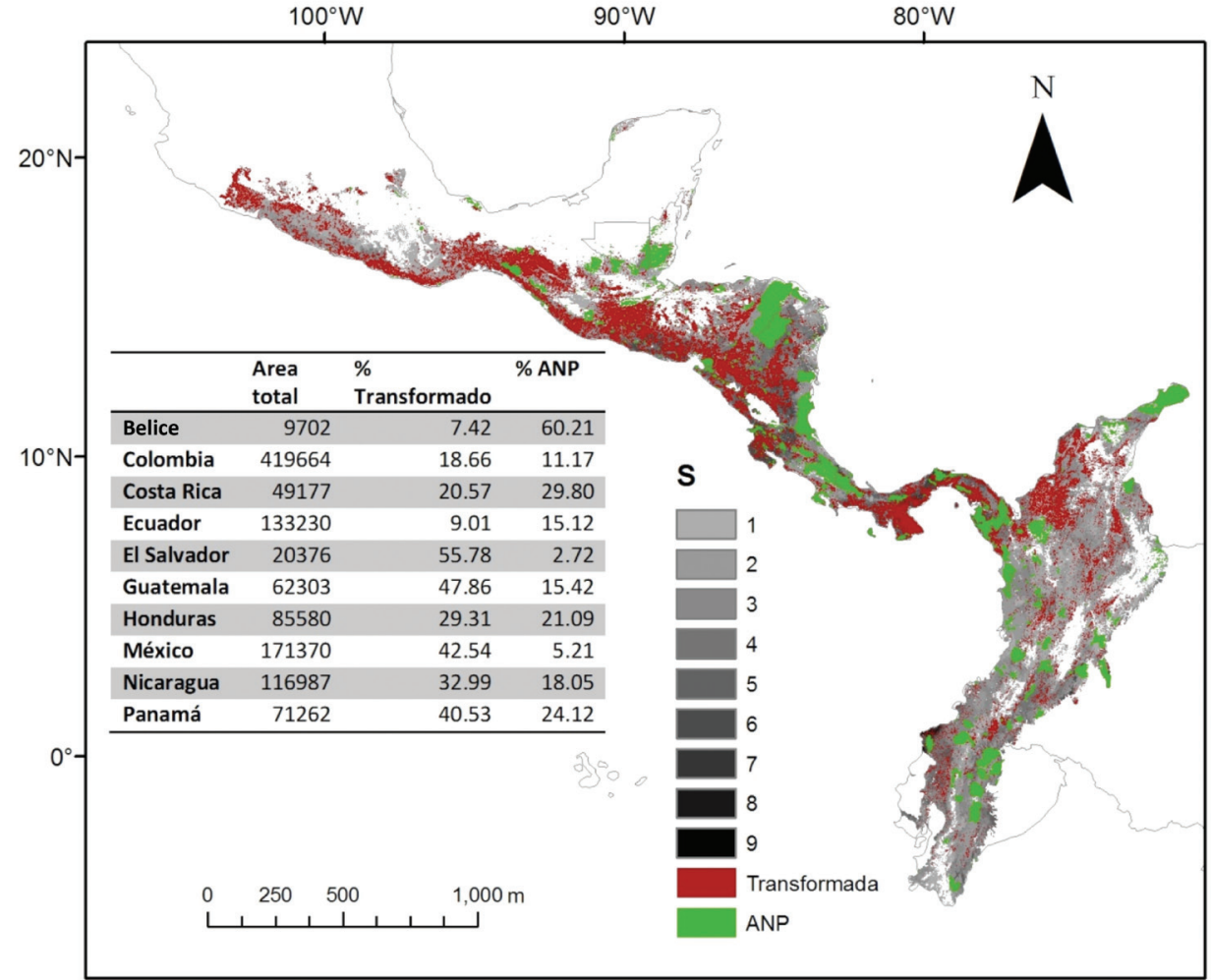


g

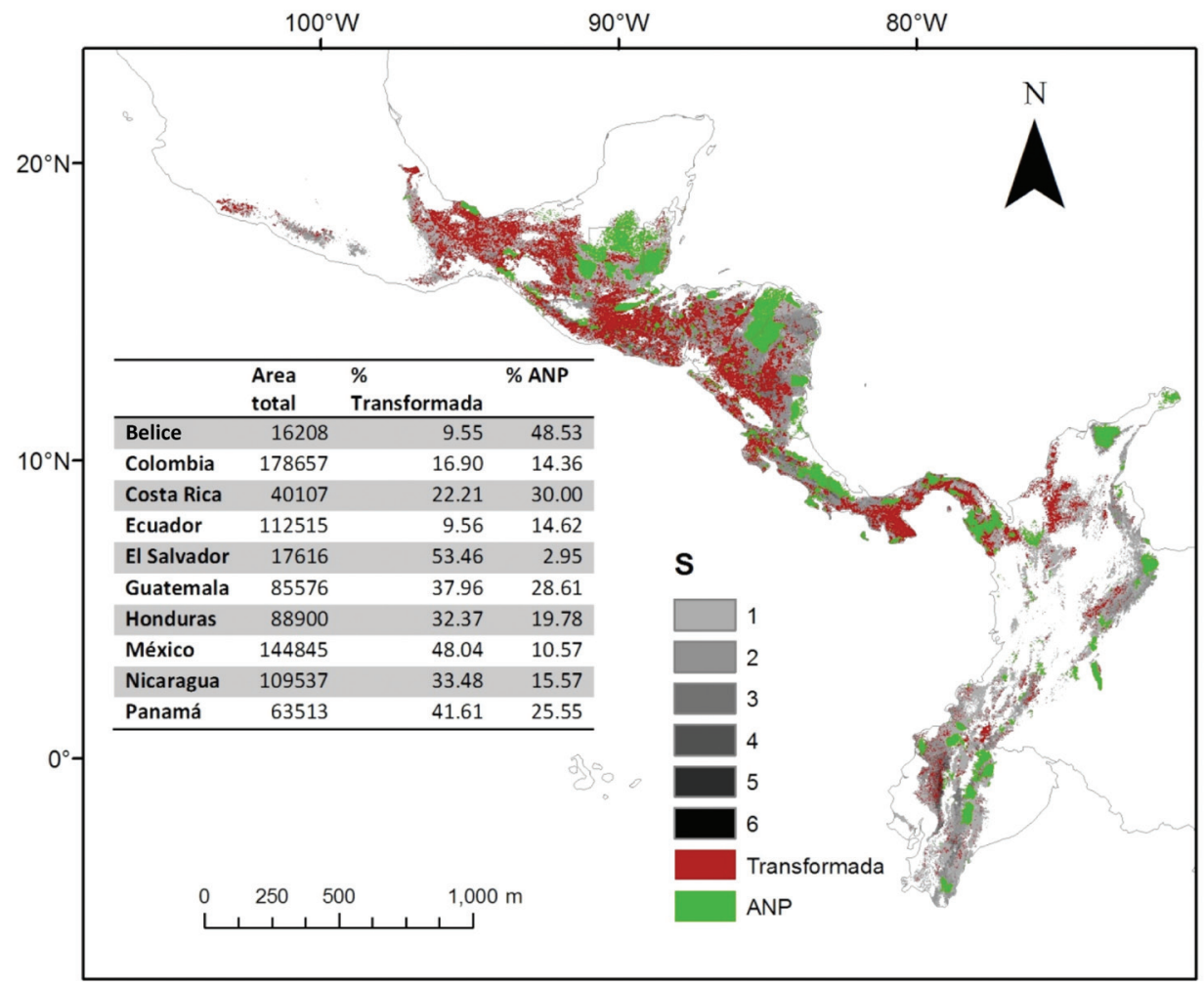

h

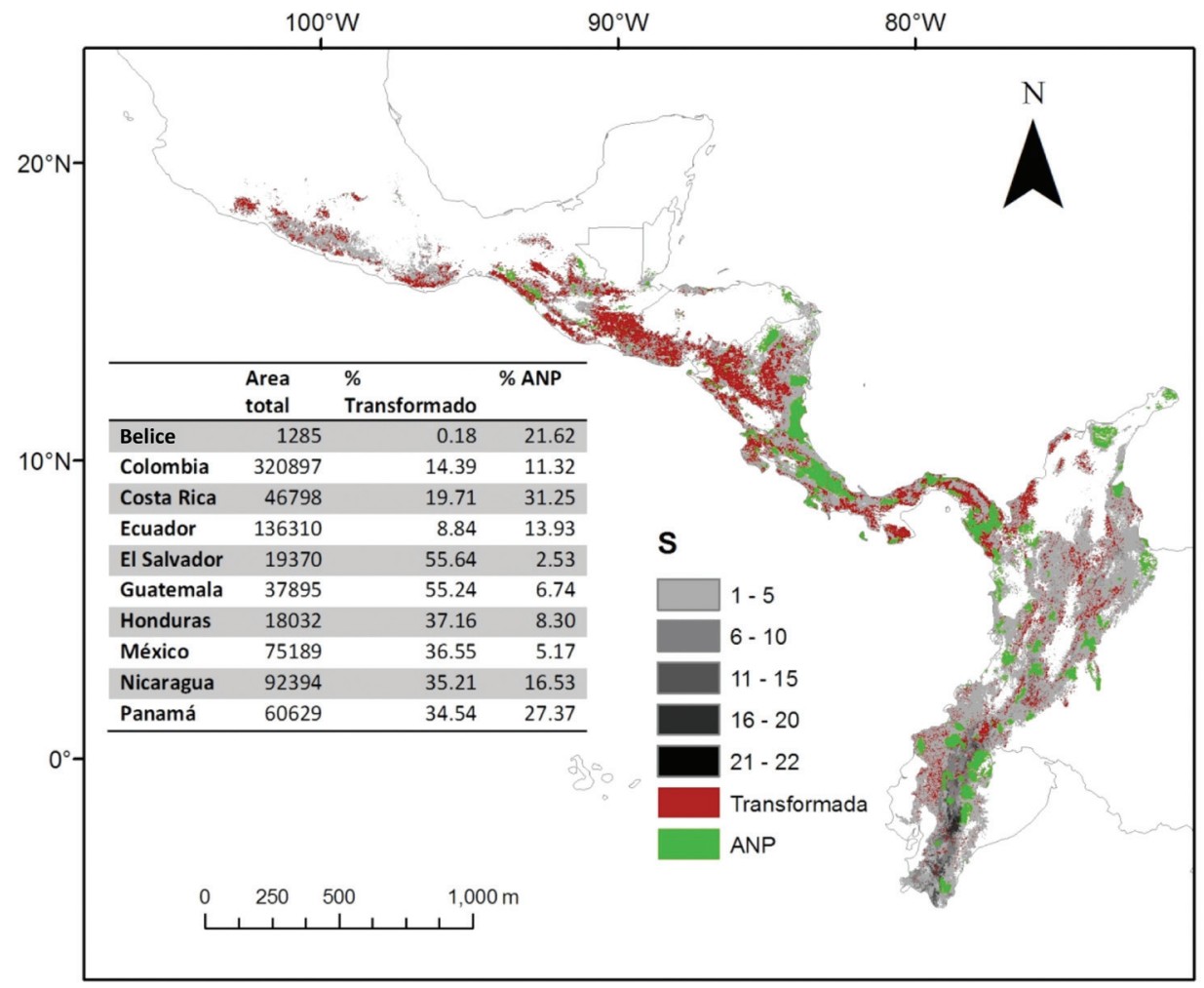



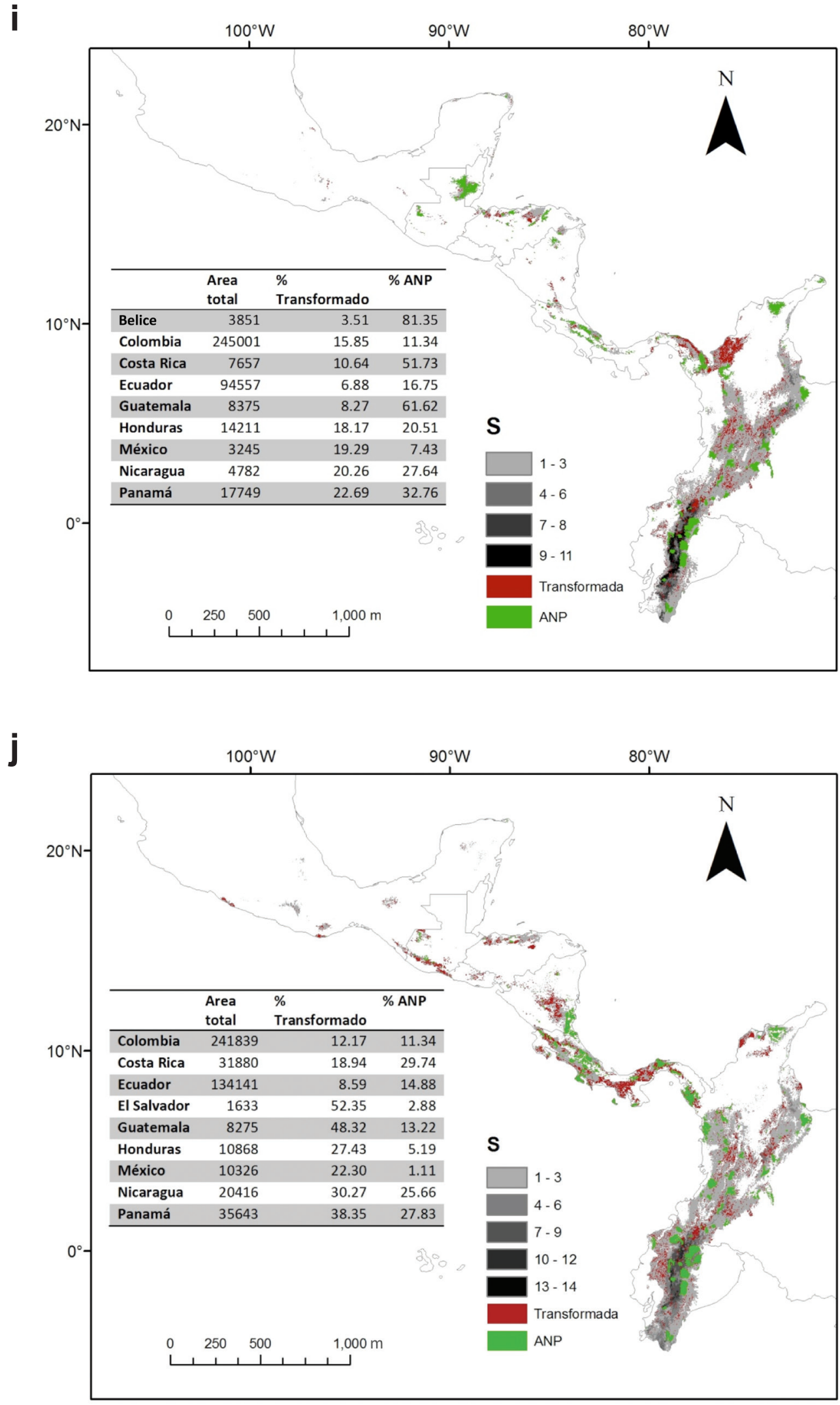


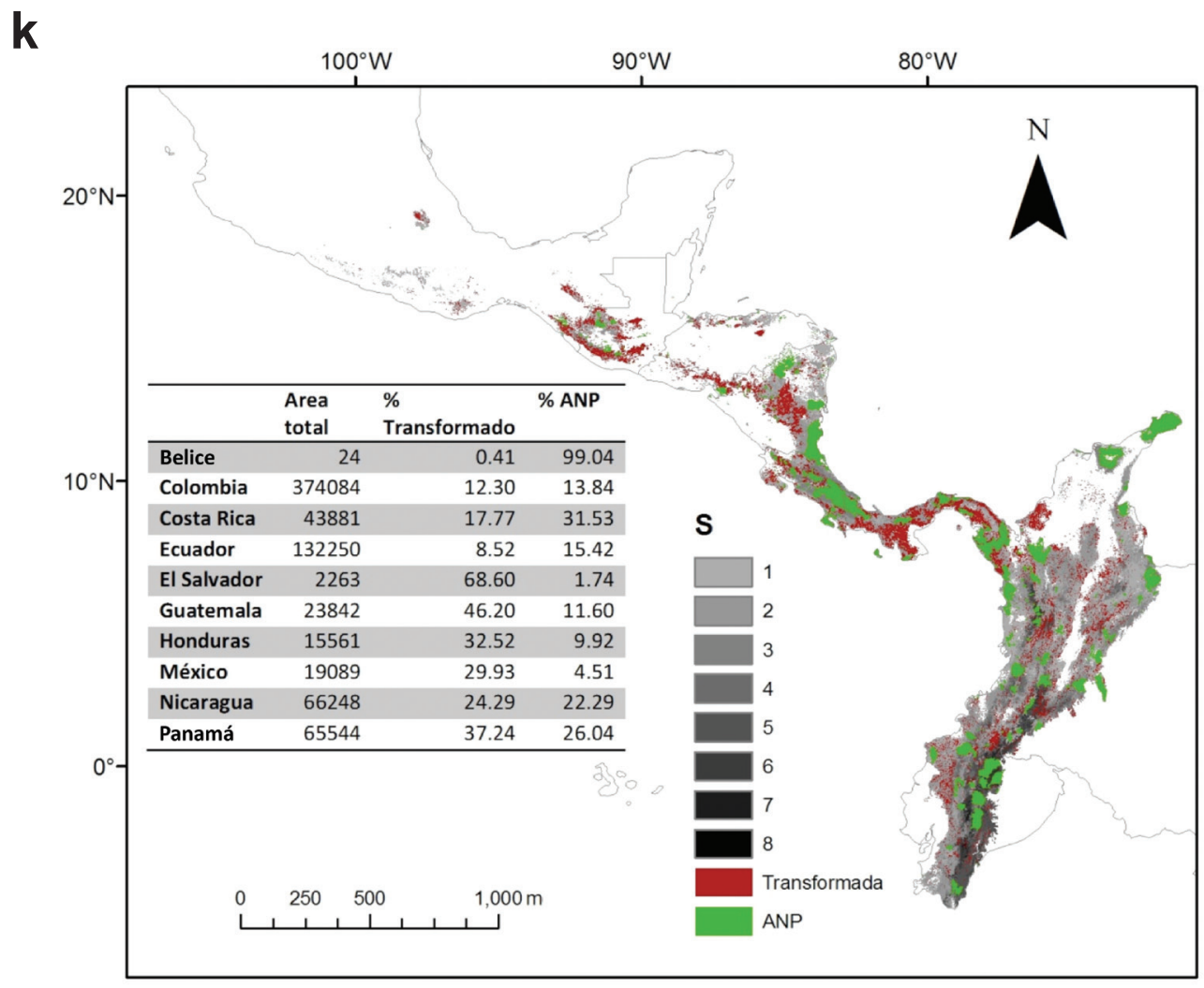

